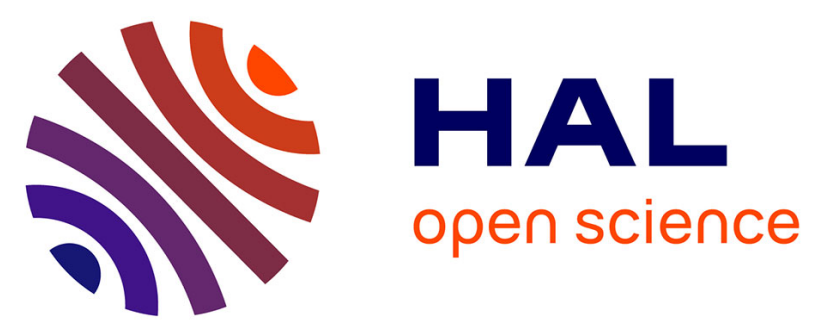

\title{
Application of the differential effective medium theory to determine fluid saturation and crack density from measured P- and S-wave velocities
}

Matthias Zillmer, Boris M. Kashtan, Fatou Doukoure, Jean-Michel Marthelot

\section{- To cite this version:}

Matthias Zillmer, Boris M. Kashtan, Fatou Doukoure, Jean-Michel Marthelot. Application of the differential effective medium theory to determine fluid saturation and crack density from measured Pand S-wave velocities. Geophysical Journal International, 2021, 10.1093/gji/ggab077 . hal-03225685

\section{HAL Id: hal-03225685 \\ https://hal.science/hal-03225685}

Submitted on 12 May 2021

HAL is a multi-disciplinary open access archive for the deposit and dissemination of scientific research documents, whether they are published or not. The documents may come from teaching and research institutions in France or abroad, or from public or private research centers.
L'archive ouverte pluridisciplinaire HAL, est destinée au dépôt et à la diffusion de documents scientifiques de niveau recherche, publiés ou non, émanant des établissements d'enseignement et de recherche français ou étrangers, des laboratoires publics ou privés. 


\title{
Application of the differential effective medium theory to
}

\section{determine fluid saturation and crack density from measured}

\section{$P$ and $S$ wave velocities}

\author{
M. Zillmer ${ }^{1}$, B.M. Kashtan ${ }^{2}$, F. Doukoure ${ }^{1}$ and J.-M. Marthelot ${ }^{1}$ \\ ${ }^{1}$ Institut Terre et Environnement de Strasbourg, Université de Strasbourg, EOST, CNRS, UMR 7063, 5 rue René Descartes, \\ F-67084 Strasbourg Cedex, France,E-mail: matthias.zillmer@unistra.fr \\ ${ }^{2}$ Institute for Physics, St Petersburg State University, Ulyanovskaya 1, Petrodvorets, St Petersburg 198904, Russia
}

\section{SUMMARY}

The differential effective medium (DEM) theory studied in this article describes elastic moduli of a fractured medium with help of differential equations, where crack density is the independent variable and fluid saturation is a parameter. The effective medium is isotropic for randomly oriented flat ellipsoidal cracks and thus fully characterized by two elastic constants. In this article we derive an analytical solution of the equation for Poisson's ratio and we transform the differential equation for Young's modulus into a nonlinear algebraic equation. Fluid saturation and crack density can then be determined from measured wave propágation velocities by a simple algorithm. We also derive approximate solutions for elastic moduli as a function of crack density and saturation, which allows to quantify the uncertainty of the result due to measurement errors. The DEM theory leads to higher crack densities than the self-consistent (SC) method and to lower crack densities than the non-interacting (NI) theory for measured elastic moduli, while all three methods give similar fluid saturation fractions. As an example of application of our theoretical results, we study weathered granite in the Strengbach water catchment in the Vosges mountains in France. We have performed full waveform sonic logging measurements 
in an $86 \mathrm{~m}$ deep borehole located at an altitude of $1130 \mathrm{~m}$ above sea level, which is used for hydro-geophysical and geochemical studies of a granitic aquifer. The logging data allows us to investigate $P$ and $S$ waves in the depth range between 40 and $80 \mathrm{~m}$. The $P$ and $S$ wave propagation velocities take average values of $5.0 \mathrm{~km} / \mathrm{s}$ and $2.7 \mathrm{~km} / \mathrm{s}$, respectively, with the highest values of $5.8 \mathrm{~km} / \mathrm{s}$ and $3.2 \mathrm{~km} / \mathrm{s}$ at $75-80 \mathrm{~m}$ depth. From these velocities we obtain a water saturation of $75 \pm 25 \%$. The crack density describes the degree of weathering of the granite, which generally decreases with depth, but takes high values near layers of strongly weathered granite. Crack density is on average 0.5, with the highest value of 1.0 at $65 \mathrm{~m}$ and the lowest value of 0.2 at $75 \mathrm{~m}$ depth. The analysis of the full waveform logging data by the DEM method supports results from previous geochemical and hydrological studies in the Strengbach catchment which concluded that water is stored in deeper layers of the granitic aquifer.

Key words: Acoustic properties; Body waves; Downhole methods; Hydrogeophysics; Wave propagation.

\section{Introduction}

The effect of a stress field on a single crack in a 3D elastic medium was first investigated by Sack (1946), Sneddon (1946), Segedin (1951) and Eshelby (1957) who calculated the change in elastic strain energy. The presence of many small cracks reduces the elastic moduli of a material and correspondingly the velocities of compressional and shear waves which propagate through the cracked medium. In fracture mechanics and geophysics 'static' effective medium theories for inclusions and cracks were developed for example by Bristow (1960); Walsh (1965); Salganik (1973); O'Connell \& Budiansky (1974); Budiansky \& O’Connell (1976); Bruner (1976); Kachanov (1980); Henyey \& Pomphrey (1982); Kémeny \& Cook (1986). Scattering theory was used to obtain 'dynamic' effective moduli (Chatterjee \& Knopoff 1978; Hudson 1980). The wavelength is supposed to be large compared to crack size and crack spacing, so that the resulting fractured medium can be described as a homogeneous effective medium. Another fracture model is based on parallel slip interfaces (Schoenberg \& Douma 1988).

Elastic moduli as a function of crack density differ considerably between effective medium theories, even for methods which are based on the same single crack model. For the 'self-consistent' (SC) method of O'Connell \& Budiansky (1974) and Budiansky \& O'Connell (1976) the elastic moduli 
become zero at a finite crack density. Bruner (1976) and Henyey \& Pomphrey (1982) have emphasized that the potential energy should be calculated by an incremental way. For this 'differential effective medium' (DEM) method or 'differential self-consistent' method the elastic moduli are zero only at infinite crack density. This is also the case for Kachanov's 'non-interacting' (NI) crack theory (Kachanov 1980, 1992). The figures in Sayers \& Kachanov (1991) show that for small crack densities the DEM method leads to static moduli which are close to the dynamic moduli obtained by Hudson (1980) with scattering theory. The DEM moduli remain bounded for high crack density when the solution by second order scattering theory diverges and thus fails. Computer simulations were performed with Finite Element and Finite Difference methods to simulate wave propagation in 2D and 3D cracked media. Seismic velocities of these simulations were then compared to effective medium theories and often the numerical results were explained best by the DEM theory (Dahm \& Becker 1998; Saenger \& Shapiro 2002; Saenger, Krüger \& Shapiro 2004; Vasylevskyi, Drach \& Tsukrov 2018). DEM results for Young's modulus are close to the result obtained by the effective field method of Levin et al. (2004) for a 2D crack problem (see also Kanaun \& Levin (2008)). Berryman, Pride \& Wang (2002) calculated approximate solutions to the coupled DEM equations for the shear and the bulk modulus.

The DEM, SC and NI crack theories were applied to various problems in seismology, rock physics and near surface geophysics. O'Connell \& Budiansky (1974) developed the SC crack theory and used it to interpret observed seismic velocity variations before the 1971 San Fernando earthquake in terms of changes in crack density and saturation. Zhao \& Mizùno (1999) and Mishra \& Zhao (2003) estimated crack density and saturation rate in the Kobe and Bhuj earthquake areas from seismic velocities obtained by travel time tomography. Bressan et al. (2016) did the same for the Friuli region and compared the resulting saturation and crack density maps with seismicity. Popp \& Kern (1994) compared modeled velocities of low-porosity erystalline rocks with ultrasound experiments on samples from the deep drilling site KTB in Germany. Reuschlé et al. (2003) used Kachanov's NI theory to model ultrasound P-wave velocities in thermally cracked granite. Adelinet et al. (2011a) calculated dry elastic moduli for Biot-Frenkel-Gassmann's poroelastic theory with the goal to characterize the fluid state of the Earth's crust beneath Iceland. Holbrook et al. (2019) used the DEM theory to model fracture density of weathered granitic gneiss in the Appalachian Mountains. We follow their approach in our application example, but first we derive a solution to the DEM equations for the inverse problem. Our theoretical results simplify the application of the DEM to measured data for estimating crack density and fluid saturation fraction.

We consider the model of an isotropic elastic background medium with two sets of isolated flat ellipsoidal cracks which are randomly oriented, one set of cracks is completely saturated with an 
incompressible fluid (wet cracks) while the other cracks are empty (dry cracks). The non-linear differential equation for Poisson's ratio decouples and can be solved in closed form for crack density. We obtain an algebraic equation for Young's modulus which depends on fluid saturation fraction. We also derive approximate solutions for the elastic moduli as a function of crack density and fluid saturation. These equations can be used for modeling instead of the differential equations and they show how measurement errors influence the accuracy of the obtained results.

We apply the theoretical method to experimental data from full waveform sonic logging measurements. We recorded the borehole data in the Strengbach water catchment area in the Vosges mountains in Alsace (France). $P$ and $S$ wave velocities are converted to crack density and water saturation. As a result we quantify the degree of weathering of the granite in the borehole between 40 and $80 \mathrm{~m}$ depth.

\section{Solution of the elastic DEM equations}

Solution for $\epsilon$ and $E(\xi), \mu(\xi), K(\xi)$

Bruner (1976) derived the following differential equations for Poisson's ratio $\nu$ and Young's modulus $E$ with crack density $\epsilon$ as the independent variable:

$$
\begin{aligned}
& \frac{d \nu}{d \epsilon}=-\frac{16}{45} \frac{1-\nu^{2}}{2-\nu}\left[(1-\xi)\left(2+5 \nu-3 \nu^{2}\right)-2(1-2 \nu)\right], \\
& \frac{1}{E} \frac{d E}{d \epsilon}=-\frac{16}{45} \frac{1-\nu^{2}}{2-\nu}[3(1-\xi)(2-\nu)+4] .
\end{aligned}
$$

For $N$ flat ellipsoidal cracks in a volume $V$ the crack density is defined by $\epsilon=\frac{N}{V}\left\langle a^{3}\right\rangle$ where $a$ is the larger half-axis of the ellipsoid. The brackets denote an average over different crack sizes. The cracks are randomly oriented, so that the effective medium is isotropic and fully described by two elastic moduli. The parameter $\xi$, where $0 \leq \xi \leq 1$, denotes the fraction of (wet) cracks saturated by an incompressible fluid, while $1-\xi$ is the fraction of unsaturated (dry) cracks. Salganik (1973) has derived these differential equations first for the special case of dry cracks $(\xi=0)$. Equations 1 and 2 can be solved numerically with initial values $\nu(\epsilon=0) \equiv \nu_{0}$ and $E(\epsilon=0) \equiv E_{0}$ for an unfractured medium. An exact solution in closed form can be calculated for the case that all cracks are saturated, $\xi=1$ (appendix A).

The crack density $\epsilon$ does not appear explicitly on the right hand side (rhs) of eq. 1 for the Poisson's ratio, so that this non-linear equation decouples and can be solved by separation of variables (Courant $\&$ John 1974). The details are given in appendix B.

$$
\begin{gathered}
\epsilon\left(\xi, \nu_{0}, \nu\right)=\frac{45}{64} \frac{1}{3-2 \xi} \ln \frac{1-\nu}{1-\nu_{0}}+\frac{45}{64} \frac{1}{2-\xi} \ln \frac{1+\nu}{1+\nu_{0}}-\frac{45}{128} \frac{5-3 \xi}{(2-\xi)(3-2 \xi)} \ln \frac{3(1-\xi) \nu^{2}-(9-5 \xi) \nu+2 \xi}{3(1-\xi) \nu_{0}^{2}-(9-5 \xi) \nu_{0}+2 \xi} \\
+\frac{45}{128} \frac{(9-5 \xi)(7-5 \xi)-4(1-\xi)(3-\xi)}{(2-\xi)(3-2 \xi) w(\xi)} \ln \frac{6(1-\xi) \nu \nu_{0}-(9-5 \xi)\left(\nu+\nu_{0}\right)+w(\xi)\left(\nu-\nu_{0}\right)+4 \xi}{6(1-\xi) \nu_{0}-(9-5 \xi)\left(\nu+\nu_{0}\right)-w(\xi)\left(\nu-\nu_{0}\right)+4 \xi}
\end{gathered}
$$


where

$$
w(\xi)=\sqrt{(9-5 \xi)^{2}-24 \xi(1-\xi)} .
$$

By substituting $\frac{d \nu}{d \epsilon}$ of eq. 1 in the rhs of eq. 2 crack density is eliminated from the equations. We obtain the following algebraic equation for Young's modulus as a function of saturation $\xi$ (appendix B):

$$
\frac{E}{E_{0}}\left(\xi, \nu_{0}, \nu\right)=\left[\frac{9-5 \xi+w(\xi)-6(1-\xi) \nu}{9-5 \xi+w(\xi)-6(1-\xi) \nu_{0}}\right]^{\frac{w(\xi)-11+7 \xi}{2 w(\xi)}}\left[\frac{9-5 \xi-w(\xi)-6(1-\xi) \nu}{9-5 \xi-w(\xi)-6(1-\xi) \nu_{0}}\right]^{\frac{w(\xi)+11-7 \xi}{2 w(\xi)}}
$$

Instead of the differential equations 1 and 2 we now have the more simple equations 3 and 5; see also Case (1984), Zimmerman (1985) and Hashin (1988) for the special case of dry cracks $(\xi=0)$.

Suppose we measure the elastic constants $\nu_{0}, E_{0}$ of the unfractured medium and $\nu, E$ of the fractured medium and we want to determine crack density $\epsilon$ and saturation $\xi$. The saturation is obtained from eq. 5 by $1 \mathrm{D}$ numerical root searching and the crack density is then given directly by eq. 3 . We demonstrate in appendix B that there is a unique solution for $\xi$ which fulfills eq. 5.

Differential equations for the shear modulus $\mu$ and the bulk modulus $K$ can be easily derived from eqs. 1 and 2 by differentiating the standard relations between elastic moduli for isotropic media:

$$
\begin{aligned}
& \frac{1}{\mu} \frac{d \mu}{d \epsilon}=-\frac{32}{45} \frac{1-\nu}{2-\nu}[3+(1-\xi)(2-\nu)], \\
& \frac{1}{K} \frac{d K}{d \epsilon}=-\frac{16}{9}(1-\xi) \frac{1-\nu^{2}}{1-2 \nu} .
\end{aligned}
$$

If we use $\frac{d \nu}{d \epsilon}$ to eliminate crack density from eq. 6 or 7 then we obtain the same rhs as in eq. 5 and as a multiplicative factor the usual functions of Poisson's ratio which transform elastic moduli from $E$ to $\mu$ and from $E$ to $K$.

A differential equation for the Lamé parameter $\lambda$ does not have the same simple form. Henyey \& Pomphrey (1982) derived that equation for the limiting cases of fully saturated $(\xi=1)$ and dry $(\xi=0)$ cracks.

\section{Solution for approximated $\epsilon$ and $\nu$}

The Poisson's ratio $\nu(\epsilon)$ is required on the rhs of eqs. 2, 6 and 7 to obtain a solution for the elastic moduli as a function of crack density. Since eq. 3 cannot be solved explicitly for $\nu$, we look for an approximation and follow Bruner (1976) who noticed that $\frac{1-\nu^{2}}{2-\nu}$ varies by less than $7 \%$ in the interval $0 \leq \nu<\frac{1}{2}$. We approximate the factor by $\frac{1}{2}$ to obtain the following equation:

$$
\frac{d \nu}{d \epsilon}=-\frac{8}{45}\left[(1-\xi)\left(2+5 \nu-3 \nu^{2}\right)-2(1-2 \nu)\right] \text {. }
$$

This Riceati equation can be solved with a variable transformation (Ince 1926):

$$
\nu\left(\epsilon, \xi, \nu_{0}\right)=\frac{\left[4 \xi-\nu_{0}(9-5 \xi)\right]\left(1-e^{-\frac{8}{45} w(\xi) \epsilon}\right)+\nu_{0} w(\xi)\left(1+e^{-\frac{8}{45} w(\xi) \epsilon}\right)}{\left[9-5 \xi-\nu_{0} 6(1-\xi)\right]\left(1-e^{-\frac{8}{45} w(\xi) \epsilon}\right)+w(\xi)\left(1+e^{-\frac{8}{45} w(\xi) \epsilon}\right)}
$$


where $w(\xi)$ is given by eq. 4 .

We can solve eq. 9 for $\epsilon(\nu)$ or eq. 8 by separation of variables which leads to:

$$
\epsilon\left(\xi, \nu, \nu_{0}\right)=\frac{45}{8 w(\xi)} \ln \frac{6(1-\xi) \nu \nu_{0}-(9-5 \xi)\left(\nu+\nu_{0}\right)+w(\xi)\left(\nu-\nu_{0}\right)+4 \xi}{6(1-\xi) \nu \nu_{0}-(9-5 \xi)\left(\nu+\nu_{0}\right)-w(\xi)\left(\nu-\nu_{0}\right)+4 \xi} .
$$

Eq. 10 has the form of the fourth term of eq. 3 with a slightly different coefficient.

Substituting eq. 9 on the rhs of eqs. 2, 6 and 7 does not lead to a solution by elementary integrals. Therefore we further approximate the differential equation 8 by neglecting the term proportional to $\nu^{2}$ on the rhs:

$$
\frac{d \nu}{d \epsilon}=\frac{8}{45}[2 \xi-(9-5 \xi) \nu]
$$

The solution of this equation is given by

$$
\begin{aligned}
\epsilon\left(\xi, \nu, \nu_{0}\right) & =\frac{45}{8(9-5 \xi)} \ln \frac{2 \xi-(9-5 \xi) \nu_{0}}{2 \xi-(9-5 \xi) \nu}, \\
\nu\left(\epsilon, \xi, \nu_{0}\right) & =\frac{2 \xi-\left[2 \xi-\nu_{0}(9-5 \xi)\right] e^{-\frac{8}{45}(9-5 \xi) \epsilon}}{9-5 \xi} .
\end{aligned}
$$

Note that neglecting the term proportional to $\nu \nu_{0}$ on the rhs of eq. 10 and approximating $w \approx 9-5 \xi$ also leads to eq. 12 . By using $w \approx 9-5 \xi$, eq. 9 takes a similar form as eq. 13 with an additional term in the denominator.

Fig. 1 shows Poisson's ratio $\nu(\epsilon)$ as a function of crack density.

The 1D root searching solution of eq. 3 is to numerical precision identical to the solution of the differential equation 1 by a Runge-Kutta method. The two approximations of $\nu(\epsilon)$, eqs. 9 and 13, work very well in the interval $0 \leq \nu, \nu_{0}<\frac{1}{2}$. The formulas also work for a certain range of negative Poisson's ratios but this is not important for geophysical applications.

Solution for approximated $E, \mu$ and $K$

If we substitute $\nu(\epsilon)$ of eq. 13 on the rhs of eq. 2 and use the method of separation of variables, then we obtain elementary integrals with exponential functions in the integrand, which can be solved in closed form (Gradstein \& Ryshik 1981). This leads to the following approximate solutions, where the moduli are functions of crack density $\epsilon$ and fluid saturation $\xi$. They also depend on the Poisson's ratio $\nu_{0}$ of the unfractured medium.

$$
\begin{gathered}
\ln \frac{E}{E_{0}}\left(\epsilon, \xi, \nu_{0}\right) \approx \begin{array}{c}
-\frac{16}{45}\left[\frac{2(9-5 \xi)}{3(3-2 \xi)}+3(1-\xi)\right]\left[1-\left(\frac{2 \xi}{9-5 \xi}\right)^{2}\right] \epsilon \\
+\frac{8 y_{0}}{(9-5 \xi)^{2}}\left[1-\frac{3 \xi(1-\xi)}{9-5 \xi}\right]\left[1-e^{\left.-\frac{8}{45}(9-5 \xi) \epsilon\right]}\right] \\
+\frac{3(1-\xi) y_{0}^{2}}{(9-5 \xi)^{3}}\left[1-e^{\left.-\frac{16}{45}(9-5 \xi) \epsilon\right]}+\frac{4}{3-2 \xi} \ln \frac{6(3-2 \xi)+y_{0} e^{-\frac{8}{45}(9-5 \xi) \epsilon}}{(9-5 \xi)\left(2-\nu_{0}\right)}\right.
\end{array} \\
\ln \frac{\mu}{\mu_{0}}\left(\epsilon, \xi, \nu_{0}\right) \approx
\end{gathered}
$$




$$
\begin{gathered}
-\frac{4(1-\xi) y_{0}}{(9-5 \xi)^{2}}\left[1-e^{-\frac{8}{45}(9-5 \xi) \epsilon}\right]+\frac{2}{3-2 \xi} \ln \frac{6(3-2 \xi)+y_{0} e^{-\frac{8}{45}(9-5 \xi) \epsilon}}{(9-5 \xi)\left(2-\nu_{0}\right)} \\
\ln \frac{K}{K_{0}}\left(\epsilon, \xi, \nu_{0}\right) \approx-\frac{16}{27} \frac{(9-7 \xi)(3-\xi)}{9-5 \xi} \epsilon+\frac{5(1-\xi) y_{0}}{(9-5 \xi)^{2}}\left[1-e^{-\frac{8}{45}(9-5 \xi) \epsilon}\right] \\
+\frac{5}{6} \ln \frac{(9-5 \xi)\left(1-2 \nu_{0}\right)}{9(1-\xi)+2 y_{0} e^{-\frac{8}{45}(9-5 \xi) \epsilon}}
\end{gathered}
$$

where

$$
y_{0}=2 \xi-\nu_{0}(9-5 \xi)
$$

Fig. 2 shows that the approximations for $E, \mu$ and $K$, eqs. 14-16, work very well compared to the numerical solution of the differential equations with a Runge-Kutta method.

For the $P$-wave modulus we can use

$$
\frac{K+\frac{4}{3} \mu}{K_{0}+\frac{4}{3} \mu_{0}}=\frac{1}{3} \frac{1+\nu_{0}}{1-\nu_{0}} \frac{K}{K_{0}}+\frac{2}{3} \frac{1-2 \nu_{0}}{1-\nu_{0}} \frac{\mu}{\mu_{0}}
$$

with $\frac{\mu}{\mu_{0}}$ and $\frac{K}{K_{0}}$ from eqs. 15 and 16 .

The first term of a Taylor series in crack density $\epsilon$ gives the same result for $\mu$ and for $K+\frac{4}{3} \mu$ as Hudson's scattering theory for two sets of randomly oriented cracks, a set of dry cracks which occupies the volume fraction $\xi$ and a set of wet cracks with volume fraction $1-\xi$ (Hudson 1980, 1981; Hudson $\&$ Knopoff 1989). The second order terms are not identical. For dry cracks and small crack density our approximation is also the same as Bristow's results (Bristow 1960) except for the missing denominator in Bristow's formula for $\mu$; the correct result was published by Laws \& Brockenbrough (1987).

\section{Elastic wave propagation velocities}

Since flat cracks occupy little volume, we neglect the change in bulk density. Then we obtain the reduction of the $P$ - and $S$-wave velocities $v_{p}$ and $v_{s}$ caused by the cracks from the following equations:

$$
\frac{v_{s}}{v_{s 0}} \approx \sqrt{\frac{\mu}{\mu_{0}}}, \quad \frac{v_{p}}{v_{p 0}} \approx \sqrt{\frac{K+\frac{4}{3} \mu}{K_{0}+\frac{4}{3} \mu_{0}}},
$$

where $v_{p 0}$ and $v_{s 0}$ are the velocities of the waves in the unfractured isotropic medium.

Fig. 3 illustrates this relation between the velocity reductions and crack density and saturation. With measurement errors in $v_{p}$ and $v_{s}$ it might be difficult to distinguish up to ca. $50 \%$ saturation from dry cracks. We see also that it is more difficult to determine saturation $\xi$ with high precision, if the Poisson's ratio $\nu_{0}$ of the unfractured medium is small.

\section{A second approximation for the elastic moduli}

The results of the numerical experiment in Fig. 2 show that Young's modulus is rather independant of $\nu_{0}$, which is not the case for the shear modulus $\mu$ and the bulk modulus $K$. This is caused by the 
fact that the third term in eq. 14 is small and the second and fourth terms nearly cancel each other. We write eq. 2 as

$$
\frac{1}{E} \frac{d E}{d \epsilon}=-\frac{16}{45}\left[3(1-\xi)\left(1-\nu^{2}\right)+4 \frac{1-\nu^{2}}{2-\nu}\right]
$$

and approximate $1-\nu^{2} \approx 1$ and $\frac{1-\nu^{2}}{2-\nu} \approx \frac{1}{2}$. Then we obtain the following solution for $E$ which does not depend on $\nu_{0}$ :

$$
\ln \frac{E}{E_{0}} \approx-\frac{16}{45}(5-3 \xi) \epsilon \text {. }
$$

This is a simplification of the first term in eq. 14 .

Sevostianov \& Kachanov (2019) mentioned that for dry cracks Young's modulus depends only slightly on $\nu_{0}$. Their approximate formula for $E$ can also be obtained from eq. 14 .

Since the cracked medium is isotropic, only two elastic moduli are independent. We use eqs. 21 for $E$ and eq. 13 for $\nu$ together with the standard relations between elastic moduli for isotropic media. We obtain

$$
\begin{aligned}
\frac{\mu}{\mu_{0}} & \approx \frac{\left(1+\nu_{0}\right)(9-5 \xi) e^{-\frac{16}{45}(5-3 \xi) \epsilon}}{3(3-\xi)-\left[2 \xi-\nu_{0}(9-5 \xi)\right] e^{-\frac{8}{45}(9-5 \xi) \epsilon}}, \\
\frac{K}{K_{0}} & \approx \frac{\left(1-2 \nu_{0}\right)(9-5 \xi) e^{-\frac{16}{45}(5-3 \xi) \epsilon}}{9(1-\xi)+2\left[2 \xi-\nu_{0}(9-5 \xi)\right] e^{-\frac{8}{45}(9-5 \xi) \epsilon}},
\end{aligned}
$$

and

$$
\frac{K+\frac{4}{3} \mu}{K_{0}+\frac{4}{3} \mu_{0}} \approx \frac{\mu}{\mu_{0}} \frac{1-2 \nu_{0}}{1-\nu_{0}} \frac{9-7 \xi+\left[2 \xi-\nu_{0}(9-5 \xi)\right] e^{-\frac{8}{45}(9-5 \xi) \epsilon}}{9(1-\xi)+2\left[2 \xi-\nu_{0}(9-5 \xi)\right] e^{\frac{8}{45}(9-5 \xi) \epsilon}} .
$$

Fig. 4 shows the elastic moduli of eqs. $21-23$ as a function of crack density for different saturations $\xi$. Wave propagation velocities are shown in Fig. 5, which is very similar to Fig. 3.

The preferred method to determine crack density and the fluid saturation fraction from measured data is to use eqs. 3 and 5, since these equations decouple and no approximation has been used.

\section{The SC and NI crack theories}

Like the DEM method O'Connell and Budiansky's self-consistent (SC) and Kachanov's non-interacting (NI) crack theories were derived by calculating the potential energy of a single crack in an elastic medium. The theories differ in the ways how the potential energy is calculated for many cracks. In Appendix C we write the corresponding SC and NI equations for our model of a mixture of a wet crack system and a dry crack system. In Figs. 6 and 7 we show the relation between $P$ and $S$ wave velocities and crack density and saturation for the SC and NI methods for comparison with Fig. 3 or 5 for the DEM method.

All three crack theories show a similar spread of the curves $\frac{v_{p}}{v_{p 0}}$ versus $\frac{v_{s}}{v_{s 0}}$ with saturation $\xi$, and 
this spread varies in a similar manner with Poisson's ratio $\nu_{0}$ of the unfractured medium. We conclude that for measured $P$ and $S$ wave velocities all three theories lead to similar saturations. The isolines of constant crack density are much less/more closely spaced for the SC/NI method in comparison to the DEM method, which means that the SC method gives much lower and the NI method much higher crack densities.

\section{Application of the DEM method to full waveform sonic logging data}

Full waveform acoustic logging in the Strengbach catchment

We have recorded full waveform sonic logging data in a $86 \mathrm{~m}$ deep borehole in the Vosges mountains in France on 19 July 2017. The borehole F1B is one of six boreholes in granite, which were drilled for hydro-geophysical and geochemical studies in the Strengbach water catchment (Viville et al. 2017; Pierret et al. 2018). The borehole has a diameter of $12.5 \mathrm{~cm}$ and it is not cased except for the first 8 $\mathrm{m}$. The water level in the borehole was at $4.8 \mathrm{~m}$ below the surface. We used a Mount Sopris 2SAA1000-F full waveform sonic probe with two ceramic-piezoelectric receivers at $0.9 \mathrm{~m}(\mathrm{R} 1)$ and $1.2 \mathrm{~m}$ (R2) distance from the transducer. The probe has a diameter of $4.4 \mathrm{~cm}$. A monopole source with 30 $\mathrm{kHz}$ center frequency and a bandwidth of $\pm 15 \mathrm{kHz}$ was used. The seismograms were sampled with a $1 \mu s$ time step. The measurement was repeated each $10 \mathrm{~cm}$ in depth.

The wavelength used in the logging experiment is on the decimeter scale, just like the spacing between the two receivers of the sonic probe. We assume that the granite can be described as a homogeneous effective medium on this length scale and that crack size and crack spacing are much smaller. This includes intra- and inter-granular micro-cracks generated during the formation of the granite but most importantly fracturation due to weathering (Gilkes et al. 1980; Štyriaková et al. 2012; Lybrand et al. 2019). The Brézouard granite in the area is composed of ca. $30 \%$ quartz, K-feldspar and albite, respectively, and of $5 \%$ biotite (Fichter et al. 1998). The fresh and slightly weathered granite has a low permeability of $0.2 \mathrm{mD}$ (Sausse et al. 2001).

A variable area plot of 470 data traces is shown in Fig. 8 for the depth interval between 35 and 82 $\mathrm{m}$. The $P$ head wave is the first arrival at about $250 \mu s$ (R1) and $300 \mu s(\mathrm{R} 2)$. The $S$ head wave can also be identified in this depth range, which is not the case for the first $35 \mathrm{~m}$, where the granite is highly fractured by weathering. The $S$ head wave arrives at about $400 \mu s$ (R1) and $500 \mu s$ (R2). The direct $P$ and $S$ head waves are followed by head waves, which are multiple reflected between the probe and the borehole wall.

Several depth intervals can be identified in Fig. 8 for which the head waves are delayed, most prominantly at 59-61 $\mathrm{m}$ and $62-63 \mathrm{~m}$ depth. In these two layers the rock has a sand-like quality and 
presumably a high porosity (direct observations on cores by R. Wyns, BRGM). Since the DEM theory uses the model of isolated flat cracks with low crack porosity, we are interested in the depth intervals where the granite has microfractures but is otherwise intact. High porosity layers are excluded from the following analysis. (Holbrook et al. 2019) model high-porosity layers by Biot's theory (see also Pride, S.R. (2005)).

We select a subset of the data with a very good $\mathrm{S} / \mathrm{N}$ ratio, for which it is possible to clearly identify $P$ and $S$ wave signals on both receivers. The signals should not change its wave form and period when they propagate between the two receivers. Based on these criteria only 70 of 470 traces were selected. An example of the selected data is shown in Fig. 9. The traces are muted except for one period of the $P$ and $S$ wave signals. The recorded signals have a dominant period of ca. $20 \mathrm{kHz}$. The only signal processing applied is a 4-th order Butterworth bandpass filter with $-3 \mathrm{~dB}$ points at $7 \mathrm{kHz}$ and $60 \mathrm{kHz}$ to suppress noise outside of the source signal's bandwidth.

The $P$ wave signals recorded at both receivers are cross-correlated and the same is done with the $S$ waves signals. The maxima of the cross-correlation functions give the time shifts which are about 60-70 $\mu s$ for the $P$ wave and 105-135 $\mu s$ for the $S$ wave. $P$ and $S$ wave velocities are computed dividing the receiver spacing of $30 \mathrm{~cm}$ by the time shifts. The velocities are shown in Fig. 10.

The $P$ and $S$ wave velocities follow the same general trend: they show limited variations in the depth interval from 38 to $58 \mathrm{~m}$, take lower values at $65 \mathrm{~m}$ near the two zones of highly damaged granite, are increasing between 70 and $75 \mathrm{~m}$ and remain constant between 75 and $80 \mathrm{~m}$ depth. The time shifts are determined with an accuracy of ca. $3 \mu$ s. Since the relative velocity error is the same as the relative travel time error, we obtain ca. $4 \%$ accuracy for the $P$ wave velocity and $3 \%$ for the $S$ wave velocity.

By using eqs. 5 and 3 we determine the fraction of cracks saturated with water and the crack density. Crack density as a function of depthis a mirror image of the velocity-depth functions (Fig. 10). It has a mean average of about 0.5 for the interval from 38 to $55 \mathrm{~m}$ depth, takes higher values of $0.8-1$ at $64 \mathrm{~m}$ depth and decreases to 0.3 at $80 \mathrm{~m}$ depth. Water saturation is around $75 \%$ for the whole depth range.

\section{Uncertainty of the results for crack density and saturation}

A source of error is the unknown background velocity of the unfractured granite. We have made a seismic experiment in the old silver mine 'Gabe Gottes' near Sainte-Marie-Aux-Mines on the 6 July 2016. The mine is located in a valley in the base of the mountain at an altitude of $620 \mathrm{~m}$, ca. $4 \mathrm{~km}$ to the west of borehole F1B, which is near a summit at $1130 \mathrm{~m}$ altitude. The rock in the mine is gneiss. Since it is not exposed to weathering as the granite on top of the mountain near the borehole, it should 
give us an idea about the velocities of an unfractured or less fractured rock in the same area. We used a $4.5 \mathrm{~kg}$ sledgehammer as a seismic source and a few $1 \mathrm{C}$ and $3 \mathrm{C}$ geophones in the $150 \mathrm{~m}$ long gallery of the mine. The analysis of this seismic experiment lead to a $P$ wave velocity of $6.0 \mathrm{~km} / \mathrm{s}$ and an $S$ wave velocity of 2.9-3.0 km/s (Zillmer et al. 2019). By looking at the trend of the velocities in Fig. 10 we choose $v_{p 0}=6.3 \pm 0.2 \mathrm{~km} / \mathrm{s}$ and $v_{s 0}=3.6 \mathrm{~km} / \mathrm{s} \pm 0.15 \mathrm{~km} / \mathrm{s}$ as $P$ and $S$ wave velocities of the unfractured granite.

Error propagation gives the standard deviation $\Delta$ of the relative velocity reduction caused by the cracks as

$$
\frac{\Delta\left(\frac{v}{v_{0}}\right)}{\left(\frac{v}{v_{0}}\right)}=\sqrt{\left(\frac{\Delta v}{v}\right)^{2}+\left(\frac{\Delta v_{0}}{v_{0}}\right)^{2}},
$$

where $v$ is the $P$ or $S$ velocity in the fractured medium and $v_{0}$ is the velocity in the unfractured medium. For our data the total error of the reduced velocities is then ca. $5 \%$ for both $P$ and $S$ waves.

Fig. 11 shows two selected data points. The rectangles represent errors of $5 \%$ in $\frac{v_{p}}{v_{p 0}}$ and in $\frac{v_{s}}{v_{s 0}}$. The saturation is badly constrained for low crack density, $\epsilon \approx 0.2$, where nearly all yalues between zero and one are possible. For higher crack densities, $\epsilon \approx 0.6$, the saturation is better constrained. The absolute error in crack density is rather constant, while the relative error is larger for small crack densities.

\section{Comparison with the SC and NI crack theories}

For comparison we compute fluid saturation fraction $\xi$ and crack density $\epsilon$ for our logging data also with O'Connell \& Budiansky's SC method and Kachanov's NI method (appendix C). It turns out that the saturation varies by less than $2 \%$ between the SC, NI and DEM methods. The NI theory leads to the highest and the SC theory to the lowest crack density (Fig. 12) as expected given the different decrease rates of the elastic moduli $E$ and $\mu$ with increasing crack density for the three methods (Sayers \& Kachanov 1991). The SC method might underestimate and the NI method overestimate crack density because they use the completely fractured and unfractured rock as background medium.

The close match of the saturation fraction between all three methods was surprising, so that we performed the following computer experiment: We integrated the DEM equations, eqs. 1 and 2, numerically for a set of initial values $E_{0}, \nu_{0}$ with different saturation parameters $\xi$. Then we computed the saturation with eqs. (C.5) and (C.12). The saturation of the SC method is often close to the $\xi$ parameter which was chosen to integrate the differential equations. We suggest to use this value as an initial guess for root searching with eq. 5 , if it is located in the same half-infinite interval as the root (appendix B). 


\section{Validation of results and impact on hydrological modeling}

Viville et al. (2006) measured oxygen-18 concentrations in water as an environmental tracer in the Strengbach catchment with the goal to determine water transit time and storage capacity. By using the model of Małoszewski \& Zuber (1982) they concluded that the amount of water stored in the catchment corresponds to a thick layer of granitic bedrock $(>45 \mathrm{~m})$ with an average porosity of $5 \%$. The hydrological model which successfully explained the measured oxygen-18 concentrations consists of two water reservoirs: an upper unsaturated reservoir with a short transit time and a lower groundwater reservoir. Chabaux et al. (2017) measured geochemical and isotope composition of spring water and of borehole water in the Strengbach catchment. They also concluded that water circulates differently near the surface and at depth.

Our crack density and saturation profiles for borehole F1B show that the weathered granite in the Strengbach catchment is highly fractured in the depth range from 40 to $80 \mathrm{~m}$ and that most cracks are water saturated (Fig. 10). We have identified several layers in the logging data where $P$ and $S$ wave travel times are delayed (Fig. 8), in particular the low velocity layers at 58-60 $\mathrm{m}$ and 61-62 $\mathrm{m}$ depth. These layers are potential fluid flow paths if they are not restricted to the vicinity of the borehole. Our results support the hypothesis that water is stored and can flow at depth. This information might be useful for hydrological modeling (Weil et al. 2017) and for the modeling of geochemical weathering (Goddéris et al. 2006) in the Strengbach catchment.

\section{Conclusions}

The differential equations of the elastic DEM theory are solved for the model of two sets of randomly oriented flat ellipsoidal cracks, one set of dry cracks and the second set filled with an incompressible fluid. This leads to a simple and exact numerical algorithm for the inverse problem, so that fluid saturation and crack density can be determined from measured data. Approximate formulas which connect the elastic moduli and velocities with crack density and saturation are also derived. They can be used for forward modeling and error estimation. A comparison of different methods shows that the DEM, SC and NI theories lead to similar saturation fractions for the same input data; the SC method gives the smallest and the NI method the highest crack density.

By applying the method to $P$ and $S$ wave velocities measured in full waveform acoustic logging data we have obtained crack density and saturation depth profiles for a borehole in the Strengbach catchment in the Vosges mountains in France. The weathered granite has a crack density systematically higher than 0.4 at 40 to $80 \mathrm{~m}$ depth below the surface. Second order scattering theory for wave propagation in fractured media cannot be used to analyse the logging data because the solution is di- 
vergent for such high crack densities. This justifies a posteriori the use of the DEM theory instead of scattering theory. Our results validate previous hydrological and geochemical studies which concluded that water is stored and flows at depth in the catchment.

\section{ACKNOWLEDGMENTS}

$\mathrm{MZ}$ and $\mathrm{BK}$ are responsable for the theoretical part and MZ, FD and JMM for the experimental part of this article. The work was supported by the HYDROCRIZSTO project (ANR-15-CE01-0010). Many thanks to Marie-Claire Pierret for organizing the project and to Pascal Sailhac for organizing the geophysical work package, to Daniel Viville for communicating about the Strengbach catchment, to Pierre-Daniel Matthey for technical support during the sonic logging measurement, and to Perrine Schloegel and Fally Huldrick Adji-Ondo for participating in the seismic experiment in the 'Gabe Gottes' mine. The authors thank two anonymous reviewers whose comments helped to improve the manuscript. Seismic Unix was used to process and plot the data.

\section{REFERENCES}

Adelinet, M., Dorbath, C., Le Ravalec, M., Fortin, J., \& Guéguen, Y., 2011. Deriving microstructure and fluid state within the Icelandic crust from the inversion of tomography data, Geophys. Res. Lett., 38, L03305, doi: 10.1029/2010GL046304

Berryman, J.G., Pride, S.R. \& Wang, H.F., 2002. A differential scheme for elastic properties of rocks with dry and saturated cracks, Geophysical Journal International, 151, 597-611, doi: 10.1046/j.1365246X.2002.01801.x

Brankin, R.W., Gladwell, I., \& Shampine, L.F., 1992. RKSUITE: a suite of Runge-Kutta codes for the initial value problem for ODEs, Softreport 92-S1, Department of Mathematics, Southern Methodist University, Dallas, Texas.

Bressan, G., Ponton, M., Rossi, G. \& Urban, S., 2016. Spatial organization of seismicity and fracture pattern in NE Italy and W Slovenia, J. Seismology, 20, 511-544, doi: 10.1007/s10950-015-9541-9

Bristow, J.R., 1960. Microcracks, and the static and dynamic elastic constants of annealed and heavily coldworked metals, British Journal of Applied Physics, 11, 81-85, doi: 10.1088/0508-3443/11/2/309

Bruner, W.M., 1976. Comment on 'Seismic velocities in dry and saturated cracked solids' by R.J.O'Connell and B. Budiansky, J. Geophys. Res., 81, 2573-2576, doi: 10.1029/JB081i014p02573

Budiansky, B., \& O'Connell, R.J., 1976. Elastic moduli of a cracked solid, Int. J. Solids Structures, 12, 81-97, doi: $10.1016 / 0020-7683(76) 90044-5$

Case, E.D., 1984. The effect of microcracking upon the Poisson's ratio for brittle materials, J. Mater. Sci., 19, 3702-3712, doi: 10.1007/PL00020102 
Chatterjee, A.K., Mal, A.K. \& Knopoff, L., 1978. Elastic moduli of two-component systems, J. Geophys. Res., 83, 1785-1792, doi: 10.1029/JB083iB04p01785

Chabaux, F., Viville, D., Lucas, Y, Ackerer, J., Ranchoux, C., Bosia, C., Pierret, M.C., Labasque, T., Aquilina, L., Wyns, R., Leroue, C, Dezaye, C. \& Négrel, P., 2017. Geochemical tracing and modeling of surface and deep water-rock interactions in elementary granitic watersheds (Strengbach and Ringelbach CZOs, France), Acta Geochim, 36, 363-366, doi: 10.1007/s11631-017-0163-5

Dahm, T. \& Becker, Th., 1998. On the elastic and viscous properties of media containing strongly interacting in-plane cracks, Pure appl. geophys., 151, 1-16, doi: 10.1007/s000240050102

Courant, R., \& John, F., 1974. Introduction to calculus and analysis, vol. 2, John Wiley \& Sons, New York.

Dekker, T.J., 1969. Finding a zero by means of successive linear interpolation, in Constructive Aspects of the Fundamental Theorem of Algebra, eds. B. Dejon and P. Henrici, Wiley-Interscience

Eshelby, J.D., 1957. The determination of the elastic field of an ellipsoidal inclusion, and related problems, Proc. R. Soc. Lond. A, 241, 376-396, doi: 10.1098/rspa.1957.0133

Fichter, J., Turpault, M.-P., Dambrine, E. \& Ranger, J., 1998. Mineral evolution of acid forest soils in the Strengbach catchment (Vosges mountains, N-E France), Geoderma, 82, 315-340, doi: 10.1016/S00167061(97)00107-9

Gilkes, R.J., Suddhiprakarn, A., \& Armitage, T.M., 1980. Scanning electron microscope morphology of deeply weathered granite, Clays and Clay Minerals, 28, 29-34, doi: 10.1346/CCMN.1980.0280104

Goddéris, Y., François, L.M., Probst, A., Schott, J., Moncoulon, D., Labat, D. \& Viville, D., 2006. Modelling weathering processes at the catchment scale: The WITCH numerical model, Geochim. Cosmochim. Acta, 70, 1128-1147, doi: 10.1016/j.gca.2005.11.018

Gradstein, I., \& Ryshik, I., 1981. Tables of series, products, and integrals, vol. 1, MIR. Moskow

Hashin, Z., 1988. The differential scheme and its applications to cracked materials, J. Mech. Phys. Solids, 36, 719-734, doi: 10.1016/0022-5096(88)90005-1

Henyey, F.S. \& Pomphrey, N., 1982. Self-consistent elastic moduli of a cracked solid, Geophys. Res. Lett., 9, 903-906, doi: 10.1029/GL009i008p00903

Hindmarsh, A.C., 1983. A Systematized Collection of ODE Solvers, in Scientific Computing, eds. Stepleman, R.S. et al., North-Holland, Amsterdam

Holbrook, W.S., Marcon, V., Bacon, A.R., Brantley, S.L., Carr, B.J., Flinchum, B.A., Richter, D.D. \& Riebe, C.S., 2019. Links between physical and chemical weathering inferred from a 65-m-deep borehole through Earth's critical zone, Sci Rep, 9, 4495, doi: 10.1038/s41598-019-40819-9

Hudson, J.A., 1980. Overall properties of a cracked solid, Mathematical Proceedings of the Cambridge Philosophicat Society, 88, 371-384, doi: 10.1017/S0305004100057674

Hudson, J.A. 1981. Wave speeds and attenuation of elastic waves in material containing cracks, Geophys. J. R. astr. Soc., 64, 133-150, doi: 10.1111/j.1365-246X.1981.tb02662.x

Hudson, J.A., \& Knopoff, L., 1989. Predicting the overall properties of composite materials with small-scale inclusions or cracks, PAGEOPH, 131, 551-576, doi: 10.1007/BF00876264 
Ince, E.L., Ordinary differential equations, Longmans Green and Co., London, reprint by Dover Publications, New York, 1956

Kachanov, M., 1980. Continuum model of medium with cracks, Journal of the engineering mechanics division (ASCE), 106, 1039-1051

Kachanov, M., 1992. Effective elastic properties of cracked solids: Critical review of some basic concepts, ASME. Appl. Mech. Rev., 45, 304-335, doi: 10.1115/1.3119761

Kamke, E., 1983. Differentialgleichungen, vol. 1, 10. edition, Teubner, Stuttgart

Kanaun, S.K. \& Levin, V.M., 2008. Self-consistent methods for composites, 2 volumes, Springer, Dordrecht

Kemeny, J. \& Cook, N.G.W., 1986. Effective moduli, non-linear deformation and strength of a cracked elastic solid, Int. J. Rock Mech. Min. Sci. \& Geomech. Abstr., 23, 107-118, doi: 10.1016/0148-9062(86)90337-2

Laws, N. \& Brockenbrough, J.R., 1987. The effect of micro-crack systems on the loss of stiffness of brittle solids, Int. J. Solids Structures, 23, 1247-1268, doi: 10.1016/0020-7683(87)90104-1

Levin, V., Markov, M., \& Kanaun, S., 2004. Effective field method for seismic properties of cracked rocks, J. Geophys. Res., 109, B08202, doi: 10.1029/2003JB002795

Lybrand, R.A., Austin, J.C., Fedenko, J., Gallery, R.E., Rooney, E., Schroeder, P.A., Zaharescu, D.G. \& Oafoku, O., 2019. A coupled microscopy approach to assess the nano-landscape of weathering, Sci Rep, 9, 5377, doi: 10.1038/s41598-019-41357-0

Małowszewski, P. \& Zuber, A., 1982. Determining the turnover time of groundwater systems with the aid of environmental tracers, Journal of Hydrology, 57, 207-231, doi: 10.1016/0022-1694(82)90147-0

Mishra, O.P., Zhao, D., 2003. Crack density, saturation rate and porosity at the 2001 Bhuj, India, earthquake hypocenter: a fluid-driven earthquake ?, Earth and Planetary Science Letters, 212, 393-405, doi: 10.1016/S0012-821X(03)00285-1

O’Connell, R.J. \& Budiansky, B., 1974. Seismic velocities in dry and saturated cracked solids, J. Geophys. Res., 79, 5412-5426, doi: 10.1029/JB079i035p05412

Pierret, M., Cotel, S., Ackerer, P., Beaulieu, E., Benarioumlil, S., Boucher, M., Boutin, R., Chabaux, F., Delay, F., Fourtet, C., Friedmann, P., Fritz, B., Gangloff, S., Girard, J.-F., Legtchenko, A., Viville, D., Weill, S. \& Probst, A. 2018. The Strengbach Catchment: A Multidisciplinary Environmental Sentry for 30 Years, Vadose Zone Journal, 17, 1-17, 180090, doi:10.2136/vzj2018.04.0090

Popp, T. \& Kern, H., 1994. The influence of dry and water saturated cracks on seismic velocities of crustal rocks - A comparison of experimental data with theoretical model, Surv Geophys, 15, 443-465, doi: 10.1007/BF00690169

Pride, S.R., 2005. Relationships between seismic and hydrological properties, in: Hydrogeophysics, eds. Y. Rubin and S.S. Hubbard, Springer, Dordrecht, pp. 253-290, doi: 10.1007/1-4020-3102-5_9

Reuschlé, T., Gbaguidi Haore, S., \& Darot, M., 2003. Microstructural control on the elastic properties of thermally cracked granite, Tectonophysics, 370, 95-104, doi: 10.1016/S0040-1951(03)00179-3

Sack, R.A., 1946. Extension of Griffith's theory of rupture to three dimensions, Proceedings of the Physical Society, 58, 729-736, doi: 10.1088/0959-5309/58/6/312 
Saenger, E.H. \& S.A. Shapiro, S.A., 2002. Effective velocities in fractured media: a numerical study using the rotated staggered finite-difference grid, Geophysical Prospecting, 50, 183-194, doi: 10.1046/j.13652478.2002.00309.x

Saenger, E.H., Krüger, O.S. \& Shapiro, S.A., 2004. Effective elastic properties of randomly fractured soils: 3D numerical experiments, Geophysical Prospecting, 52, 183-195, doi: 10.1111/j.1365-2478.2004.00407.x Salganik, R.L., 1973. Mechanics of bodies with many cracks, english translation of Izv. AN SSSR, Meckhanika Tverdogo Tela, 8, 149-168.

Sausse, J., Jacquot, E., Fritz, B., Leroy, J. \& Lespinasse, M., 2001. Evolution of crack permeability during fluid-rock interaction. Example of the Brézouard granite (Vosges, France), Tectonophysics, 336, 199-214, doi: 10.1016/S0040-1951(01)00102-0

Sayers, C.M. \& Kachanov, M., 1991. A simple technique for finding effective elastic constants of cracked solids for arbitray crack orientation statistics, Int. J. of Solids and Structures, 27, 671-680, doi: 10.1016/00207683(91)90027-D

Scalizzi, P., 1917. Atti Accad. Lincei, Serie 5, 26, 60-64.

Segedin, C.M., 1951. Note on a penny-shaped crack under shear, Mathematical Proceedings of the Cambridge Philosophical Society, 47, 396-400, doi: 10.1017/S0305004100026736

Schoenberg, M. \& Douma, J, 1988. Elastic wave propagation in media with parallel fractures and aligned cracks, Geophysical Prospecting, 36, 571-590, doi: 10.1111/j.1365-2478.1988.tb02181.x

Sevostianov, I. \& Kachanov, M., 2019. On the effective properties of polycrystáls with intergranular cracks, Int. J. of Solids and Structures, 156-157, 243-250, doi: 10.1016/j.ijsolstr.2018.08.017

Sneddon, I.N., 1946. The distribution of stress in the neighbourhood of a crack in an elastic solid, Proceedings of the Royal Society of London Series A, 187, 229-260, doi: 10.1098/rspa.1946.0077

Štyriaková, I., Štyriak, I. \& Oberhänsli, H., 2012. Rock weathering by indigenious heterotrophic bacteria of Bacillus spp. at different temperature: a laboratory experiment, Miner Petrol, 105, 135-144, doi: 10.1007/s00710-012-0201-2

Vasylevskyi, K., Drach, B. \& Tsukrov, 1., 2018, On micromechanical modeling of orthothropic solids with parallel cracks, Int. J. of Solids and Structures, 144-145, 46-58, doi: 10.1016/j.ijsolstr.2018.02.038

Viville, D., Ladouche, B., \& Bariac, T., 2006. Isotope hydrological study of mean transit time in the granitic Strengbach catchment (Vosges massif, France): application of the FlowPC model with modified input function, Hydrol. Process., 20, 1737-1751, doi: 10.1002/hyp.5950

Viville, D., Chabaux, F., Pierret, M.-C., Gangloff, S., Cotel, S., Probst, A., Dambrine, E., Fritz, B. \& Ambroise, B., 2017. L'Óbservatoire Hydro-Géochimique de l'Environnement (OHGE): 30 années d'observations scientifiques sur le bassin versant du Strengbach (massif des Vosges - France), Géologues, 195, 120-125.

Walsh, J.B., 1965. The effect of cracks on the compressibility of rock, J. Geophys. Res., 70, 381-389, doi: 10.1029/JZ070i002p00381

Weil, S., Delay, F., Pan, Y., Ackerer, P., 2017. A low-dimensional subsurface model for saturated and unsaturated flow processes: ability to address heterogeneity, Comput Geosci, 21, 301-314, doi: 10.1007/s10596- 
017-9613-8

Zhao, D \& Mizuno, T., 1999. Crack density and saturation rate in the 1995 Kobe earthquake region, Geophys. Res. Lett., 26, 3213-3216, doi: 10.1029/1999GL005411

Zillmer, M., Doukoure, F. \& Marthelot, J.M., 2019. Acoustic experiments in the Strengbach catchment in Alsace (France), EGU General Assembly, SM4.4, X2.335 |EGU2019-5341, Vienna

Zimmerman, R.W., 1985. The effect of microcracks on the elastic moduli of brittle materials, Journal of Materials Science Letters, 4, 1457-1460, doi: 10.1007/BF00721363 

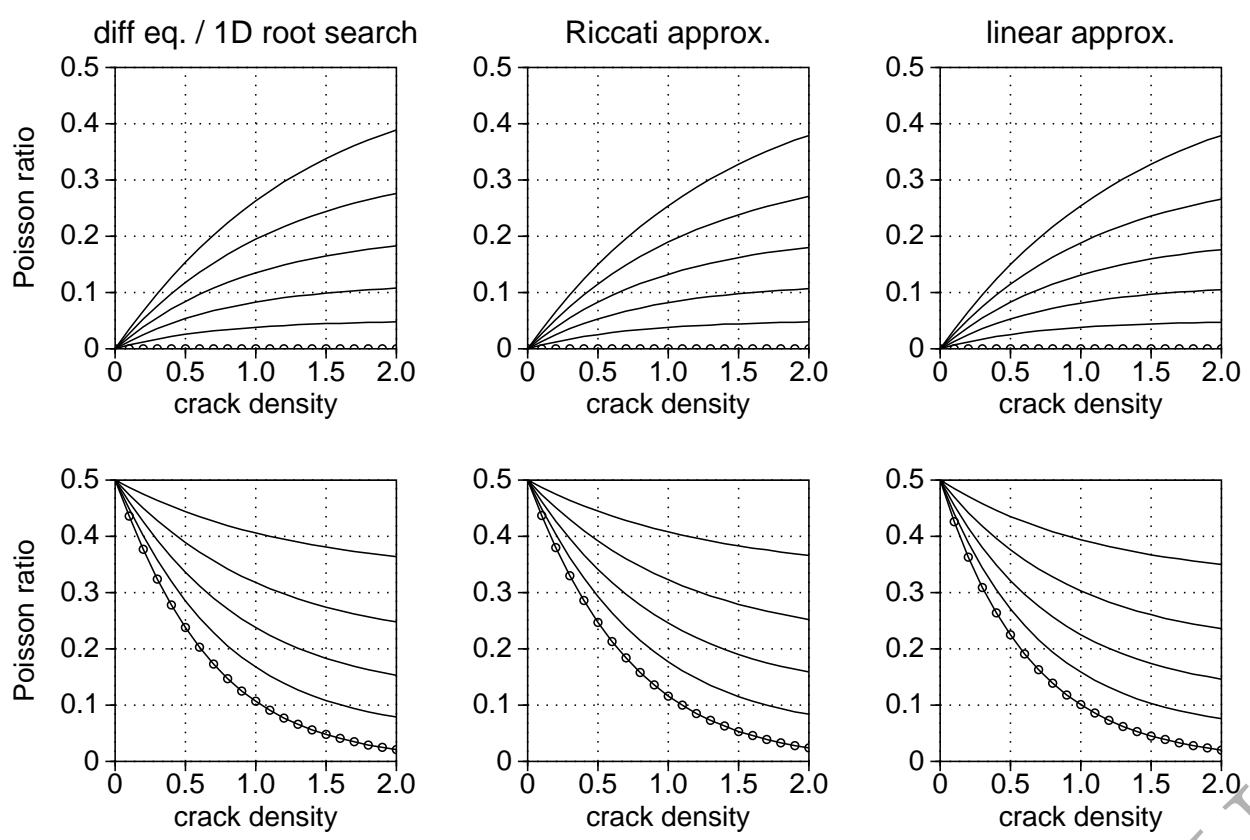

Figure 1. Poisson's ratio $\nu(\epsilon, \xi)$ : Numerical solution by eq. 1 or eq. 3 (left column) and approximations by eqs. 9 (middle column) and 13 (right column) for $\nu_{0}=0$ (top) and $\nu_{0} \rightarrow \frac{1}{2}$ (bottom). We consider various saturation values $\xi=0,0.2, \ldots, 1.0$ where $\xi=0$ is marked by open circles. $\nu \equiv \nu_{0}$ for $\xi=1, \nu_{0} \rightarrow \frac{1}{2}$. We used the program 'ut' with a Runge-Kutta $(4,5)$ formula pair as a part of the program package rksuite (Brankin, Gladwell \& Shampine 1992) for the numerical solution of the differential equations. Alternatively we also used the program 'lsode' of ODEPACK by Hindmarsh (1983). The program 'fzero' for root searching is based on a method by Dekker (1969). 

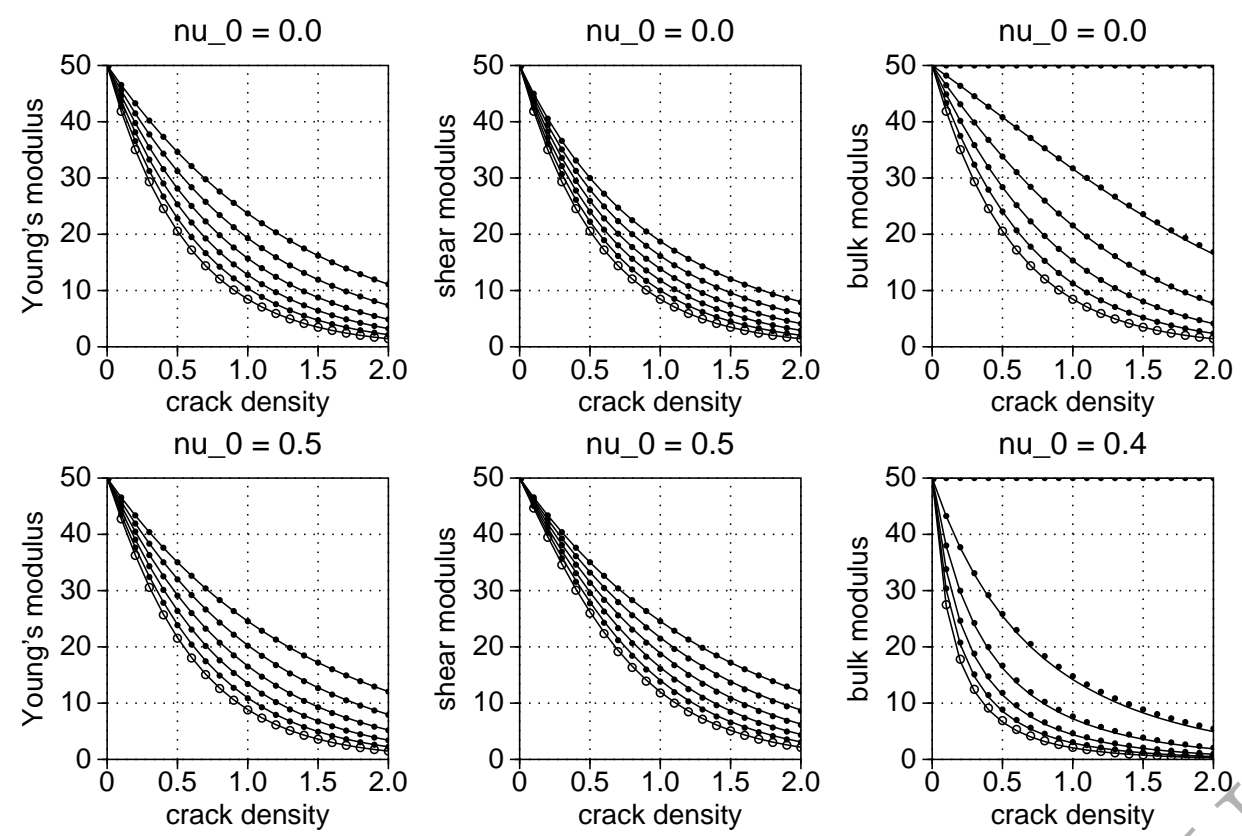

Figure 2. Young's modulus $E\left(\epsilon, \xi, \nu_{0}\right)$, shear modulus $\mu\left(\epsilon, \xi, \nu_{0}\right)$ and bulk modulus $K\left(\epsilon, \xi, \nu_{0}\right)$ for $\nu_{0}=0$ and $\nu_{0} \rightarrow \frac{1}{2}\left(\nu_{0}=0.4\right.$ for the bulk modulus). Numerical solutions (solid lines) by eqs. 2, 6 and 7 and approximations (dots) by eqs. 14, 15 and 16 are compared. Various fluid saturation values are considered: $\xi=0,0.2, \ldots, 1.0$ where $\xi=0$ is marked by open circles. 

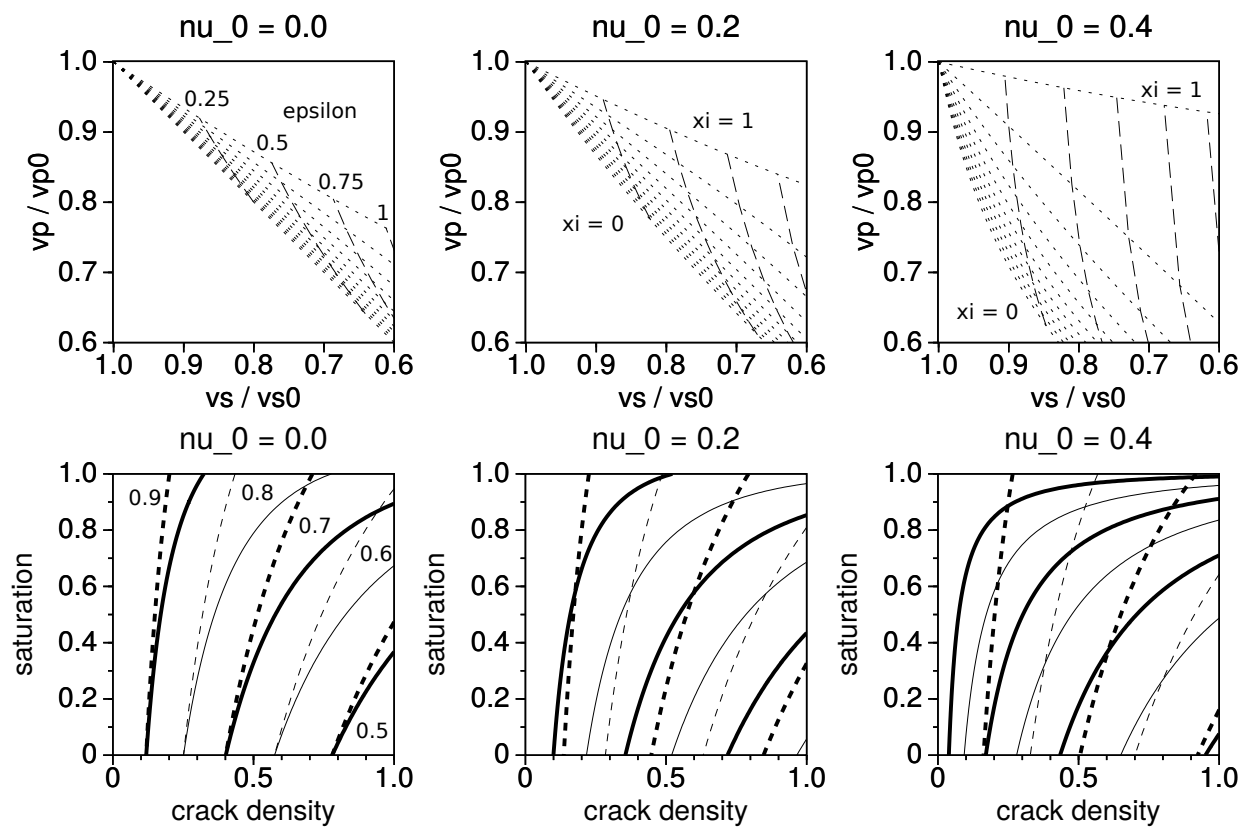

Figure 3. Top: The reduction in $P$ wave velocity $v_{p} / v_{p 0}$ and $S$ wave velocity $v_{s} / v_{s 0}$ for different values of saturation $\xi$ (dotted lines) and crack density $\epsilon$ (dashed lines). Fluid saturation takes the values $0,0.1, \ldots 1$ (from bottom to top) and crack density the values $0.25,0.5, \ldots$ (from left to right). Bottom: The reduction in $P$ wave velocity $v_{p} / v_{p 0}$ (solid lines) and $S$ wave velocity $v_{s} / v_{s 0}$ (dashed lines) for different values of saturation $\xi$ and crack density $\epsilon$. The iso-velocity lines take the values $0.9,0.8, \ldots$ (from left to right), where $0.9,0.7$ and 0.5 are printed bold. Equations 15-19 are used here. 

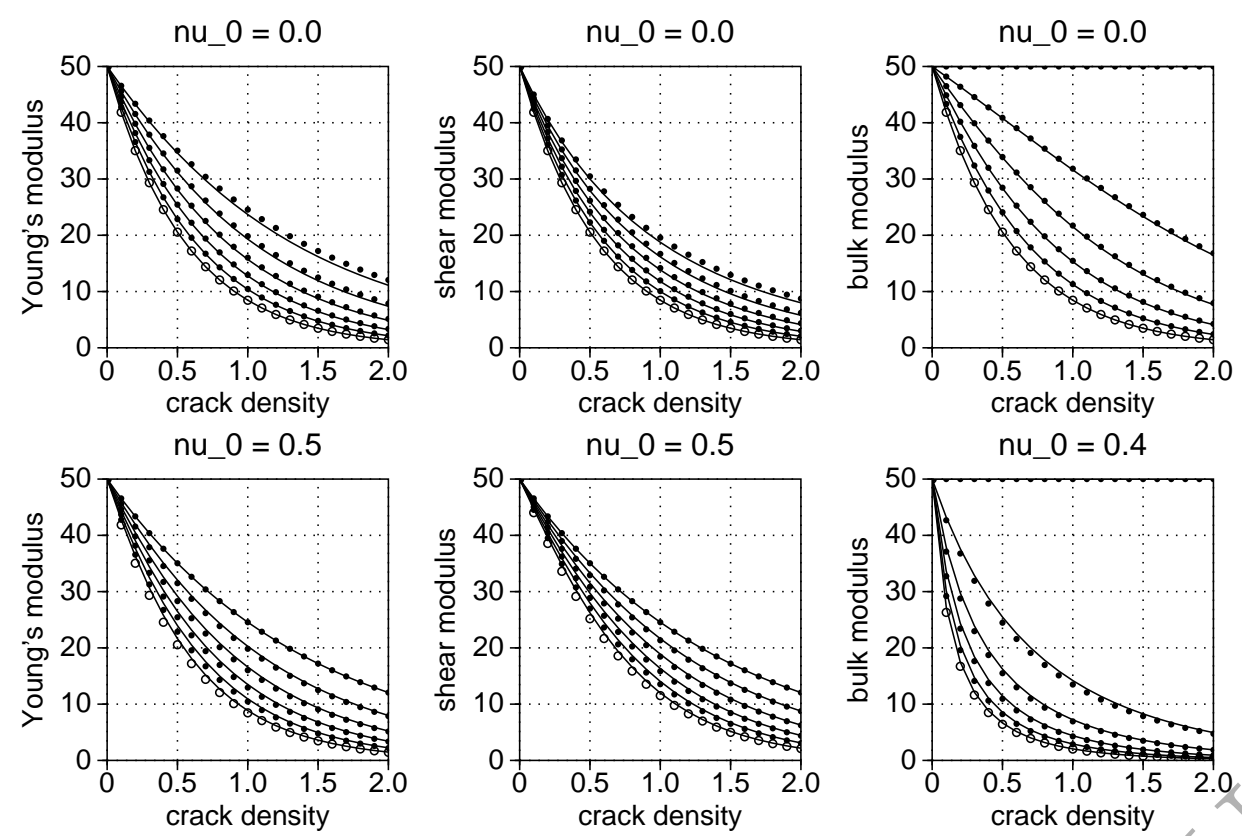

Figure 4. Young's modulus $E\left(\epsilon, \xi, \nu_{0}\right)$, shear modulus $\mu\left(\epsilon, \xi, \nu_{0}\right)$ and bulk modulus $K\left(\epsilon, \xi, \nu_{0}\right)$ for $\nu_{0}=0$ and $\nu_{0} \rightarrow \frac{1}{2}$ ( $\nu_{0}=0.4$ for the bulk modulus). Numerical solutions (solid lines) by eqs. 2, 6 and 7 and approximations (dots) by eqs. 21,22 and 23. Various fluid saturation values are considered: $\xi=0,0.2, \ldots, 1.0$ where $\xi=0$ is marked by open circles. 

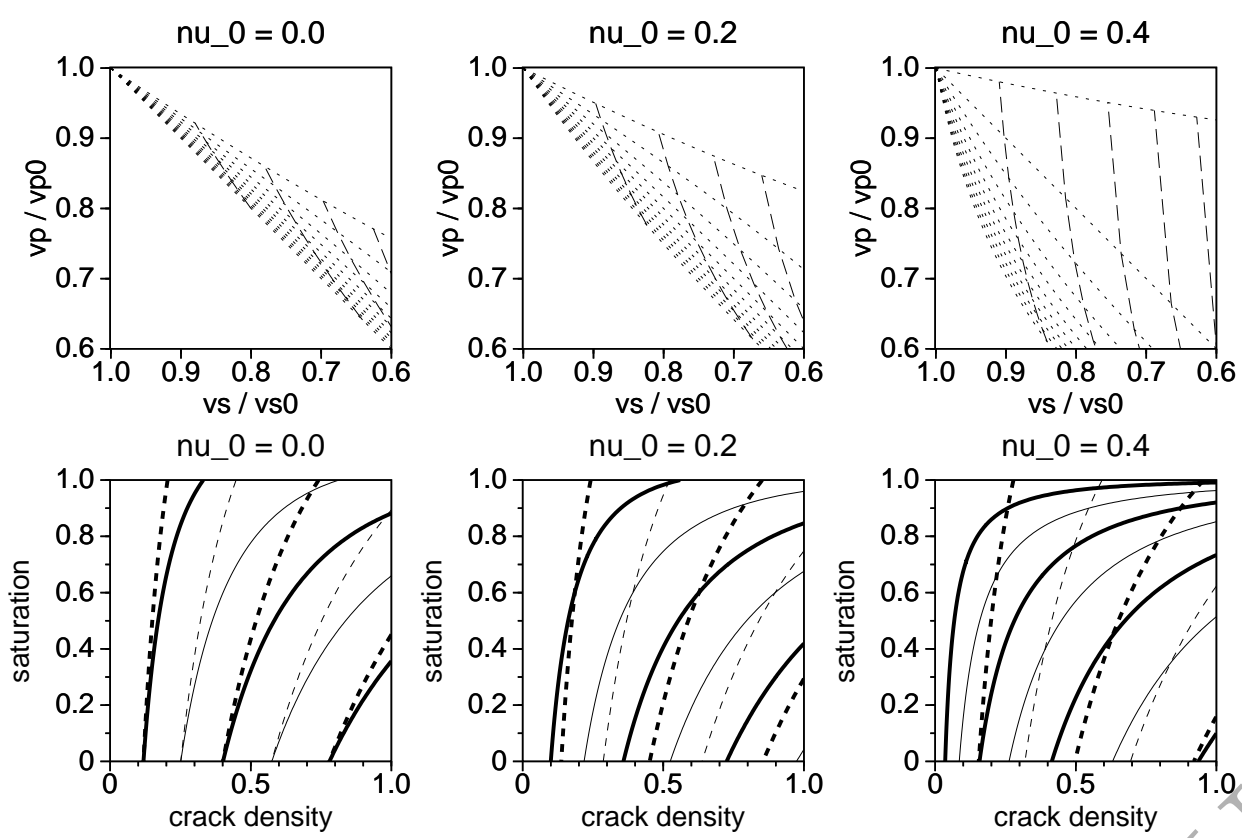

Figure 5. Same as Figure 3 but computed with eqs. 19, 22 and 24. 

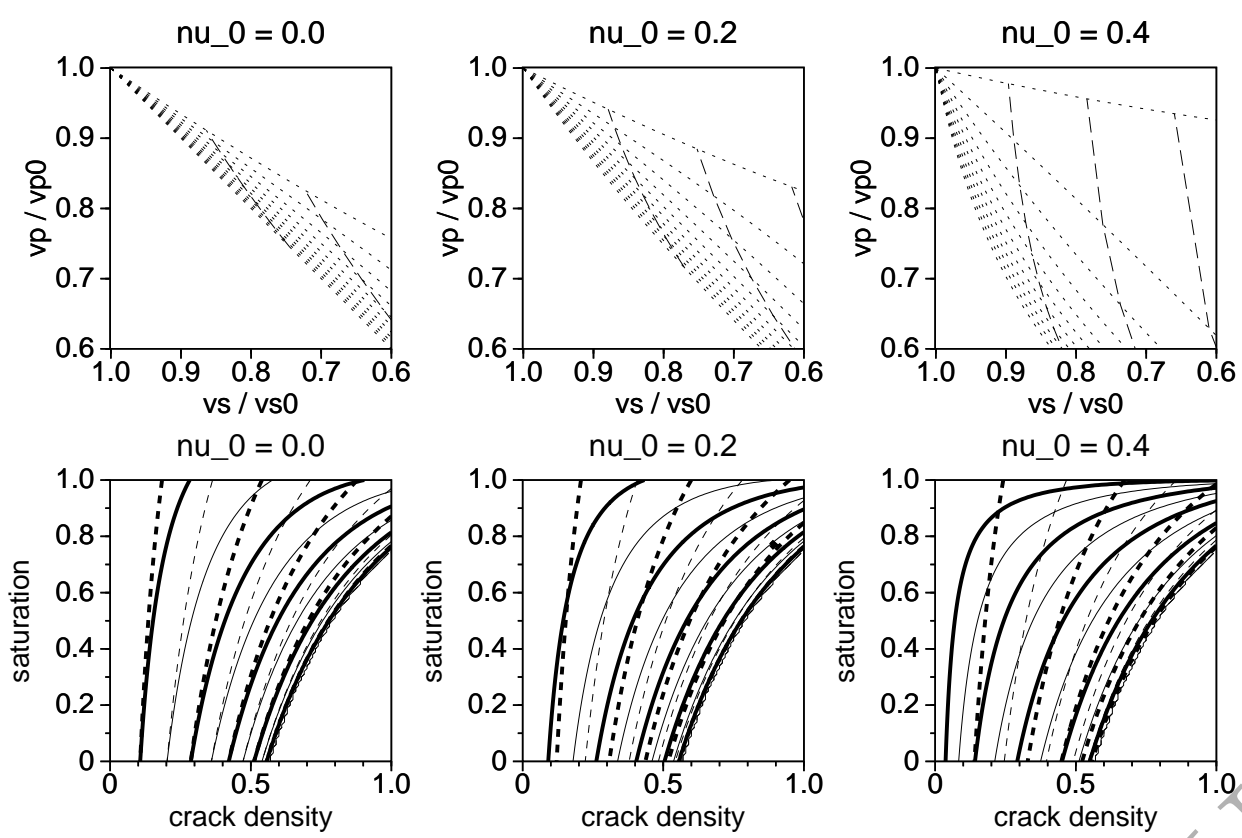

Figure 6. Same as Figure 3 but computed for O'Connell and Budiansky’s SC method. 

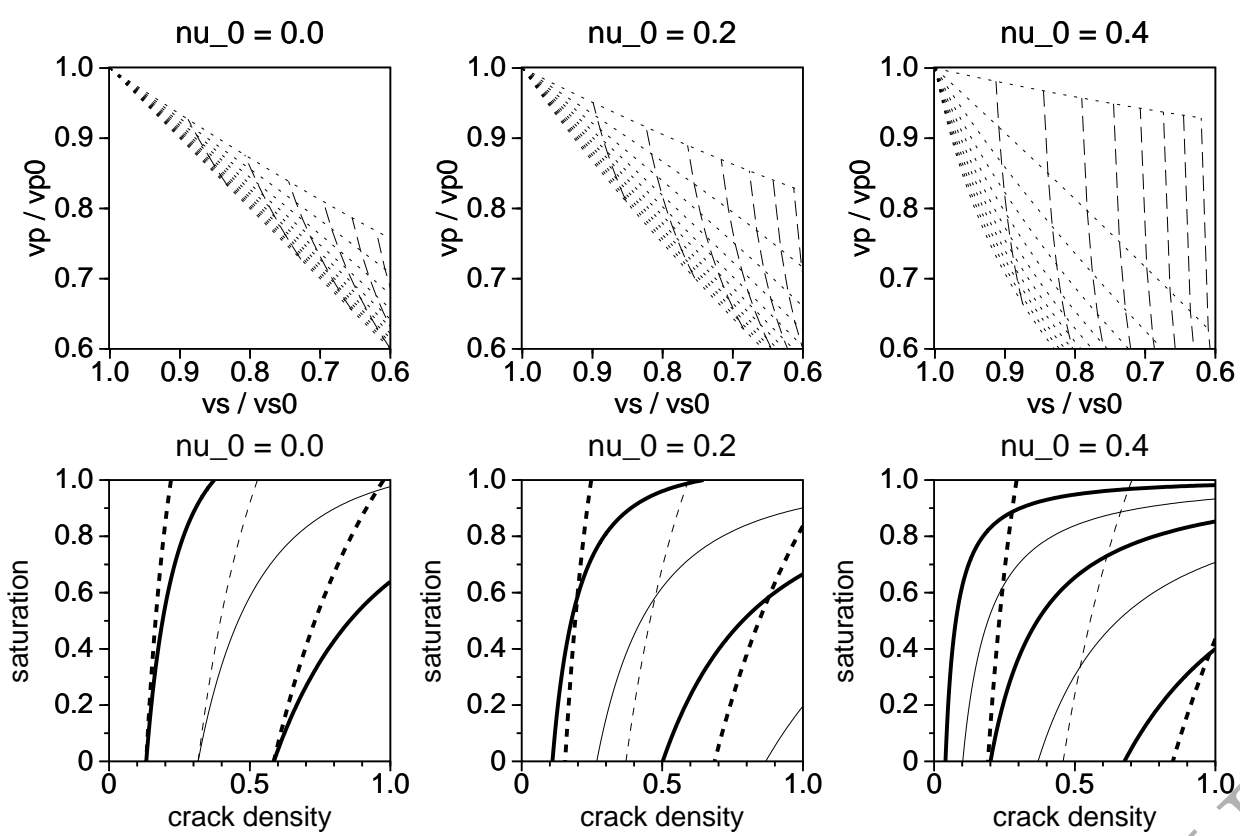

Figure 7. Same as Figure 3 but computed for Kachanov's NI method. 


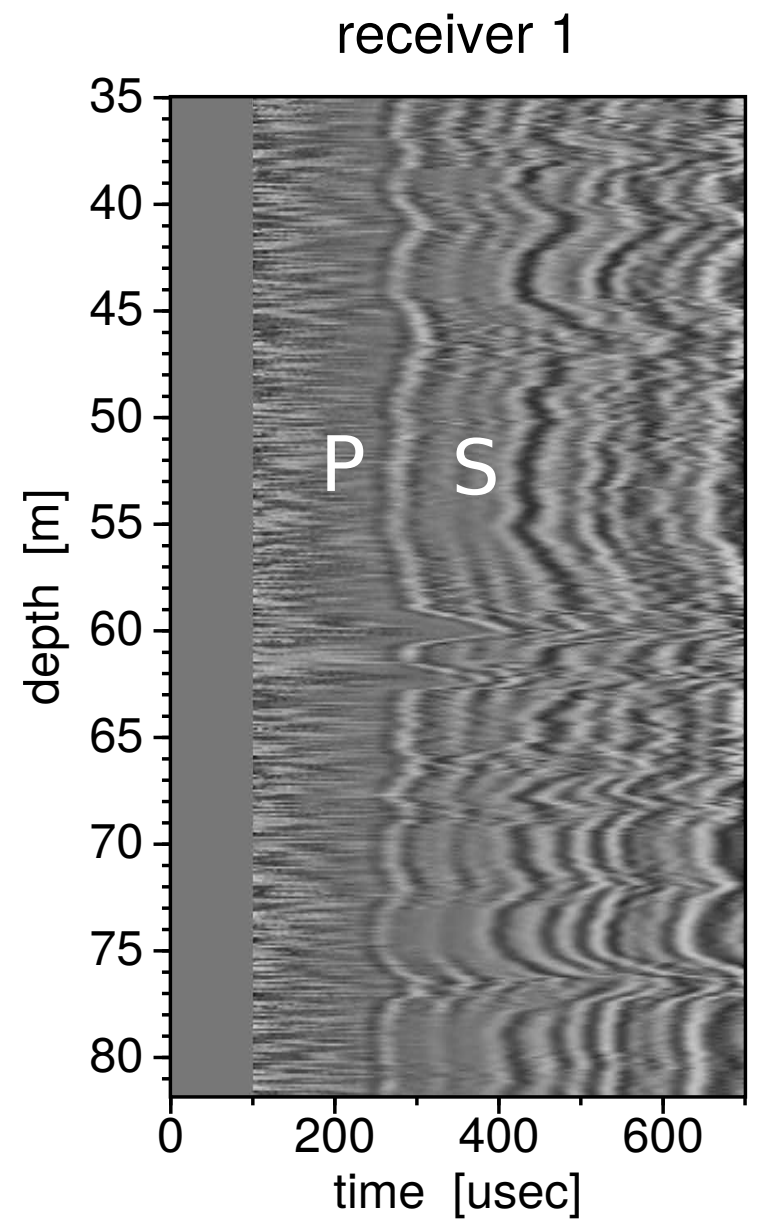

receiver 2

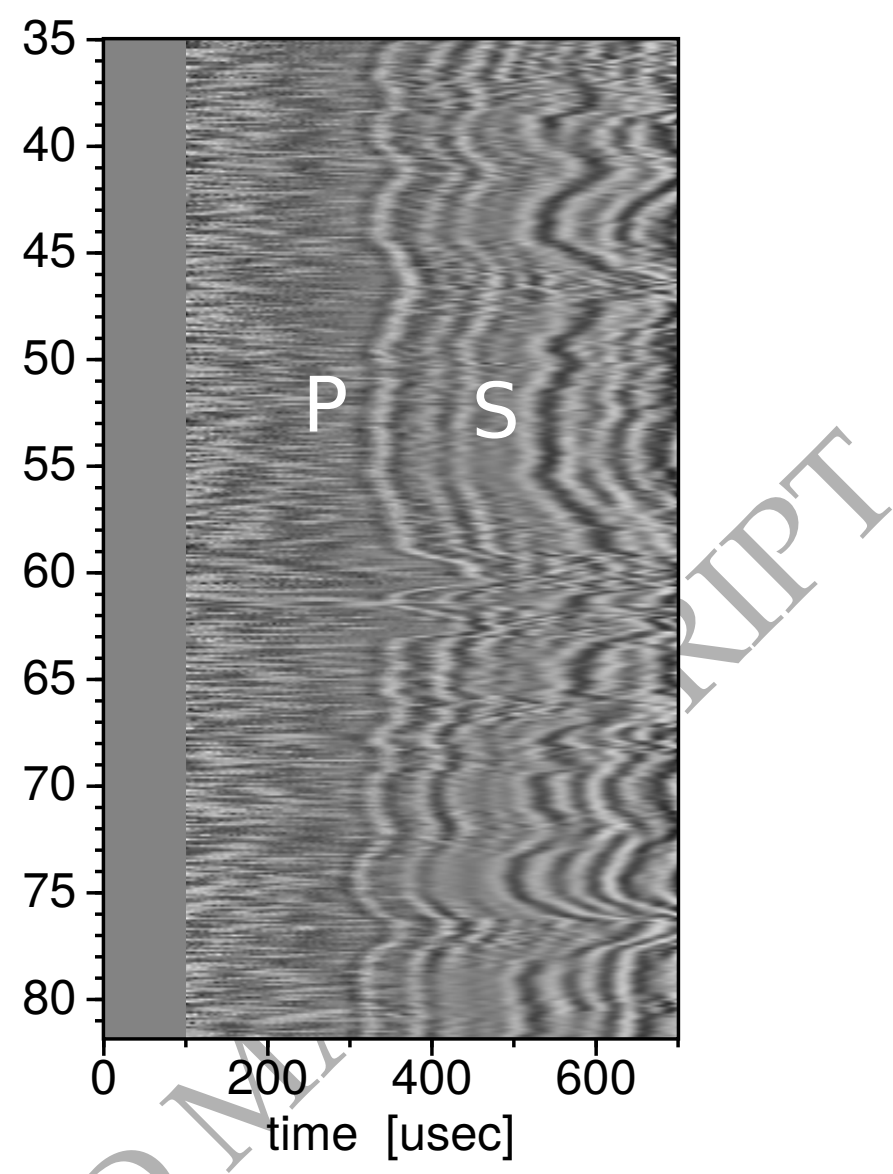

Figure 8. Full waveform sonic logging data recorded in granite in borehole F1B of the Strengbach catchment in Alsace (France) in July 2017. The distance between the piezoelectric transducer and receivers 1 and 2 is 0.9 $\mathrm{m}$ and $1.2 \mathrm{~m}$, respectively. The $\mathrm{P}$ and $\mathrm{S}$ head waves are marked. 

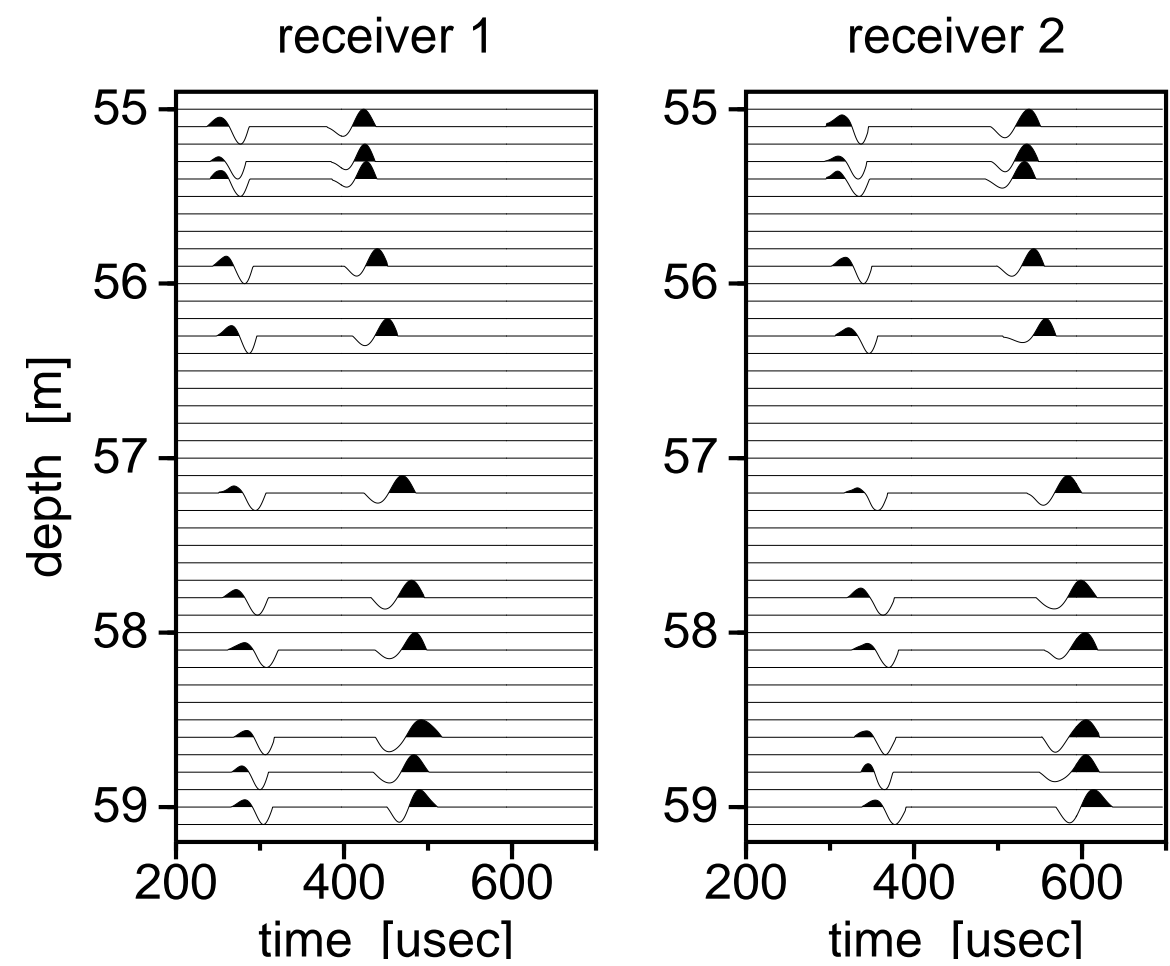

Figure 9. Example of selected $P$ and $S$ signals recorded by the two receivers. Data traces of worse quality are muted. 

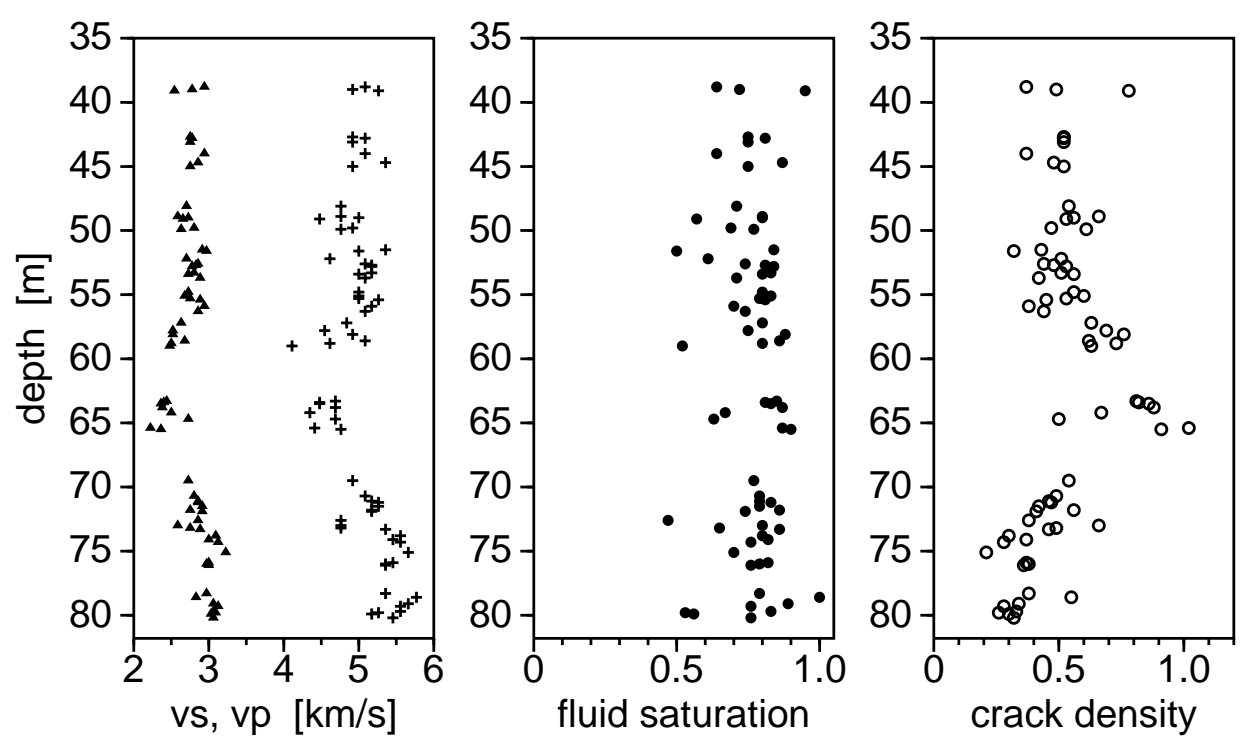

Figure 10. $P$ and $S$ wave velocities as a function of depth in borehole F1B which are obtained by analysing the travel times in the data shown in Figs. 8 and 9. The velocities (left) are used to determine the fraction of water saturated cracks from eq. 5 (middle) and crack density from eq. 3 (right). Depth intervals without data points refer to layers of strongly fractured granite where signals could not be clearly identified. We assume that the DEM model of isolated cracks can not be applied for these layers. 


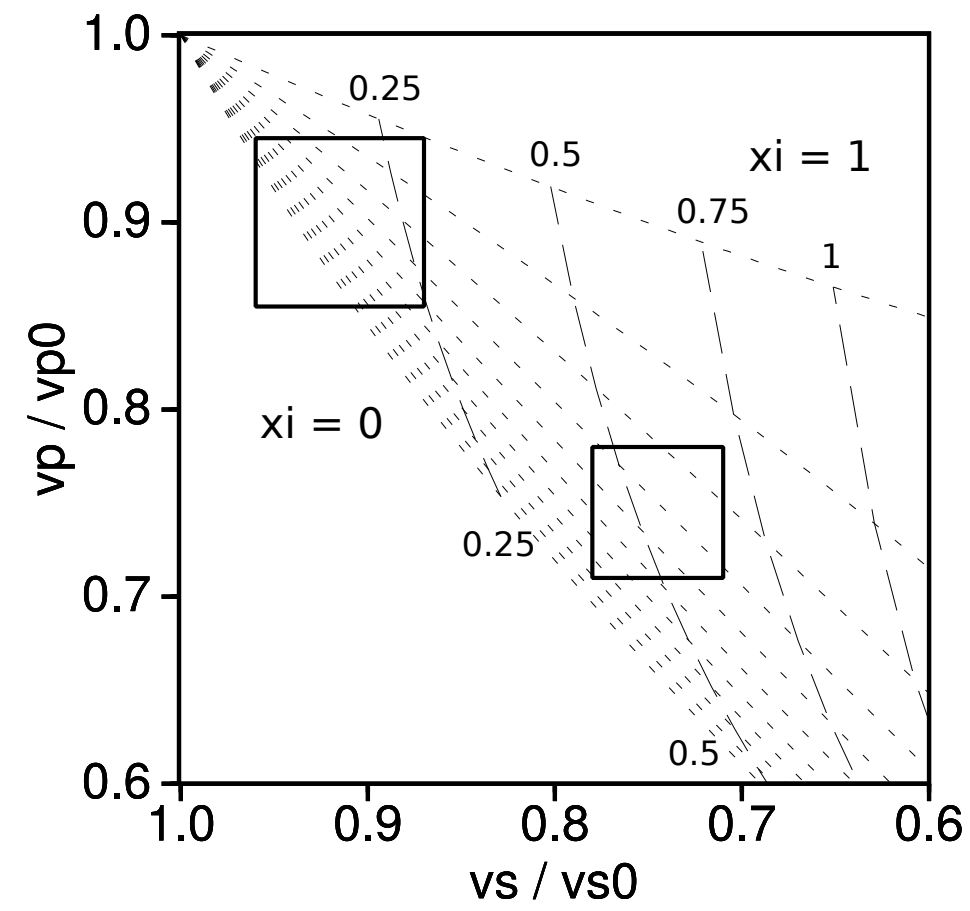

Figure 11. The uncertainty of saturation $\xi$ and crack density $\epsilon$ for $5 \%$ error in measured reduced $P$ and $S$ wave velocities. For the left data point with low crack density the saturation is nearly undetermined, while it is better constrained for the right data point at higher crack density. The Poisson's ratio of the unfractured material is $\nu_{0} \approx 0.26$. 

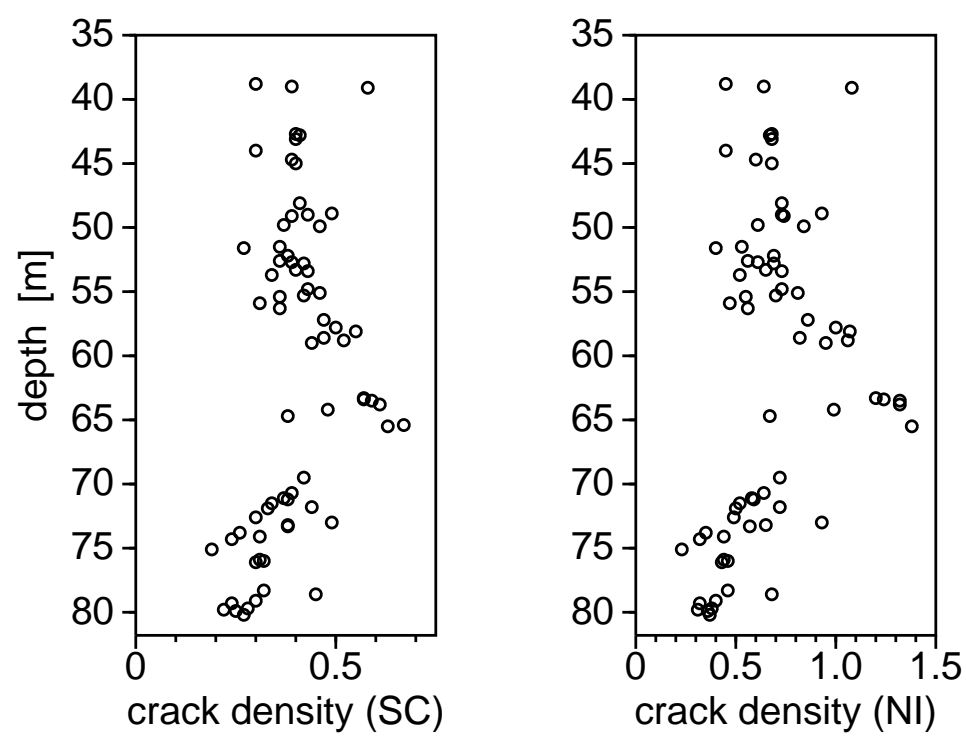

Figure 12. Crack density $\epsilon$ as a function of depth in borehole F1B in the Strengbach catchment calculated with the SC theory (eq. C.4) and with the NI theory (eq. C.11). 


\section{APPENDIX A: ELASTIC MODULI FOR MEDIA WITH SATURATED CRACKS}

For the case that all cracks are fluid saturated, $\xi=1$, the differential equation 1 for Poisson's ratio $\nu$,

$$
\frac{d \nu}{d \epsilon}=\frac{32}{45} \frac{\left(1-\nu^{2}\right)(1-2 \nu)}{2-\nu},
$$

can be solved by separation of variables and by partial fractions (Courant \& John 1974):

$$
\epsilon=\frac{45}{64} \ln \frac{1-\nu^{2}}{1-\nu_{0}^{2}}+\frac{45}{32} \ln \frac{1-2 \nu_{0}}{1-2 \nu} .
$$

This equation can be solved for $\nu(\epsilon)$. We could also transform eq. A.1 into an Abel differential equation of the first kind. It has a special relation between its coefficients and for this case a solution has been derived by Scalizzi (1917) (according to Kamke (1983)). This leads to

$$
\nu(\epsilon)=\frac{\sqrt{3\left(1-\nu_{0}^{2}\right)+\left(1-2 \nu_{0}\right)^{2} e^{-\frac{64}{45} \epsilon}}-2\left(1-2 \nu_{0}\right) e^{-\frac{32}{45} \epsilon}}{2 \sqrt{3\left(1-\nu_{0}^{2}\right)+\left(1-2 \nu_{0}\right)^{2} e^{-\frac{64}{45} \epsilon}}-\left(1-2 \nu_{0}\right) e^{-\frac{32}{45} \epsilon}} .
$$

We substitute eq. A.3 on the rhs of eq. 2 for Young's modulus $E$,

$$
\frac{1}{E} \frac{d E}{d \epsilon}=-\frac{64}{45} \frac{1-\nu^{2}}{2-\nu}
$$

to obtain

$$
\frac{E(\epsilon)}{E_{0}}=\frac{3 e^{-\frac{32}{45} \epsilon}}{2 \sqrt{3\left(1-\nu_{0}^{2}\right)+\left(1-2 \nu_{0}\right)^{2} e^{-\frac{64}{45} \epsilon}}-\left(1-2 \nu_{0}\right) e^{-\frac{32}{45} \epsilon}} .
$$

From eq. 7 follows that the bulk modulus of cracks saturated with an incompressible fluid does not change with crack density: $K \equiv K_{0}$.

We substitute eq. A.3 on the rhs of eq. 6 for the shearmodulus $\mu$,

$$
\frac{1}{\mu} \frac{d \mu}{d \epsilon}=-\frac{32}{15} \frac{1-\nu}{2-\nu}
$$

and integrate:

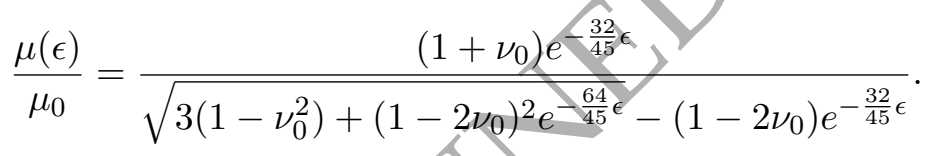

For the shear modulus the first two terms of a Taylor series in $\epsilon$ are given by

$$
\frac{\mu(\epsilon)}{\mu_{0}} \approx 1-\frac{32}{15} \frac{\lambda_{0}+2 \mu_{0}}{3 \lambda_{0}+4 \mu_{0}} \epsilon+\frac{1}{6}\left(\frac{32}{15}\right)^{2} \frac{\left(\lambda_{0}+2 \mu_{0}\right)\left(9 \lambda_{0}^{2}+36 \lambda_{0} \mu_{0}+28 \mu_{0}^{2}\right)}{\left(3 \lambda_{0}+4 \mu_{0}\right)^{3}} \epsilon^{2},
$$

where $\lambda_{0}$ and $\mu_{0}$ are the Lamé parameters of the unfractured medium.

Hudson (1980) obtained $\mu$ to second order in $\epsilon$ by using scattering theory. He noted that his result agrees with the self-consistent theory of O'Connell \& Budiansky (1974) only to first order, but not to second order. This happens again here with the DEM theory.

Henyey \& Pomphrey (1982) derived the solution for Poisson's ratio $\nu$ as a function of crack density $\epsilon$, but for the other moduli only by eliminating $\epsilon$ from the equations. A part of their result is incorrectly printed. 


\section{APPENDIX B: SOLUTION OF THE DIFFERENTIAL EQUATIONS FOR POISSON'S RATIO AND YOUNG'S MODULUS}

In this appendix we first show how to obtain the solution of the differential equation for Poisson's ratio. We write eq. 1 in the form

$$
\frac{d \nu}{d \epsilon}=\frac{16}{45} \frac{1-\nu^{2}}{2-\nu}\left[3(1-\xi) \nu^{2}-(9-5 \xi) \nu+2 \xi\right]
$$

and determine the roots $\nu_{1}, \nu_{2}$ of the quadratic polynomial on the rhs:

$$
3(1-\xi) \nu^{2}-(9-5 \xi) \nu+2 \xi=3(1-\xi)\left(\nu-\nu_{1}\right)\left(\nu-\nu_{2}\right) .
$$

We obtain

$$
\nu_{1}(\xi)=\frac{9-5 \xi+w(\xi)}{6(1-\xi)}, \quad \nu_{2}(\xi)=\frac{9-5 \xi-w(\xi)}{6(1-\xi)}
$$

where

$$
w(\xi)=\sqrt{(9-5 \xi)^{2}-24 \xi(1-\xi)} .
$$

Eq. B.1 then takes the form

$$
\frac{d \nu}{d \epsilon}=\frac{4}{135(1-\xi)} \frac{1-\nu^{2}}{2-\nu}[6(1-\xi) \nu-(9-5 \xi)+w][6(1-\xi) \nu-(9-5 \xi)-w] .
$$

By separation of variables we obtain the following integrals:

$$
\int \frac{(2-\nu)(1-\xi)}{(1+\nu)(1-\nu)[6(1-\xi) \nu-(9-5 \xi)+w][6(1-\xi) \nu-(9-5 \xi)-w]} d \nu=\frac{4}{135} \int d \epsilon \text {. }
$$

Applying the method of partial fractions to the integral on the left hand side we obtain the solution (eq. 3) after some basic calculations:

$$
\begin{gathered}
\epsilon\left(\xi, \nu, \nu_{0}\right)=\frac{45}{64} \frac{1}{3-2 \xi} \ln \frac{1-\nu}{1-\nu_{0}}+\frac{45}{64} \frac{1}{2-\xi} \ln \frac{1+\nu}{1+\nu_{0}}-\frac{45}{128} \frac{5-3 \xi}{(2-\xi)(3-2 \xi)} \ln \left[\frac{\nu-\nu_{1}(\xi)}{\nu_{0}-\nu_{1}(\xi)} \frac{\nu-\nu_{2}(\xi)}{\nu_{0}-\nu_{2}(\xi)}\right] \\
+\frac{45}{128} \frac{(9-5 \xi)(7-5 \xi)-4(1-\xi)(3-\xi)}{(2-\xi)(3-2 \xi) w(\xi)} \ln \left[\frac{\nu-\nu_{1}(\xi)}{\nu_{0}-\nu_{1}(\xi)} \frac{\nu_{0}-\nu_{2}(\xi)}{\nu-\nu_{2}(\xi)}\right] .
\end{gathered}
$$

Next, we derive an algebraic equation for Young's modulus by eliminating crack density from the differential equations. Substituting $\frac{d \nu}{d \epsilon}$ of eq. 1 in the rhs of eq. 2 gives

$$
\frac{1}{E} \frac{d E}{d \epsilon}=\frac{d \nu}{d \epsilon}(-) \frac{(2-\nu)+\frac{4}{3(1-\xi)}}{\left(\nu-\nu_{1}\right)\left(\nu-\nu_{2}\right)}
$$

where $\nu_{1}$ and $\nu_{2}$ are given by eq. B.3. By using partial fractions we obtain

$$
\begin{aligned}
& \ln \frac{E}{E_{0}}=\frac{3(1-\xi)\left(\nu_{1}-2\right)-4}{3(1-\xi)\left(\nu_{1}-\nu_{2}\right)} \ln \frac{\nu_{1}-\nu}{\nu_{1}-\nu_{0}}+\frac{3(1-\xi)\left(2-\nu_{2}\right)+4}{3(1-\xi)\left(\nu_{1}-\nu_{2}\right)} \ln \frac{\nu-\nu_{2}}{\nu_{0}-\nu_{2}}, \\
& 2 w \ln \frac{E}{E_{0}}=(w-11+7 \xi) \ln \frac{\nu_{1}-\nu}{\nu_{1}-\nu_{0}}+(w+11-7 \xi) \ln \frac{\nu-\nu_{2}}{\nu_{0}-\nu_{2}}
\end{aligned}
$$

and finally eq. 5 :

$\frac{E}{E_{0}}=\left(\frac{\nu_{1}(\xi)-\nu}{\nu_{1}(\xi)-\nu_{0}}\right)^{\frac{w(\xi)-11+7 \xi}{2 w(\xi)}}\left(\frac{\nu-\nu_{2}(\xi)}{\nu_{0}-\nu_{2}(\xi)}\right)^{\frac{w(\xi)+11-7 \xi}{2 w(\xi)}}$ 
If $\nu_{0}, \nu, E_{0}$ and $E$ are known from measurements, where $0<\frac{E}{E_{0}}<1$, then the saturation $\xi$ can be found by searching a root of $\frac{E}{E_{0}}-f(\xi)=0$, where $f$ is the function on the rhs of eq. B.11.

Let's assume for the moment that $\xi$ can take any value on the real axis. The function $f(\xi)$ has the following properties:

$$
\begin{gathered}
f(\xi \rightarrow \pm \infty) \rightarrow \frac{1+3 \nu}{1+3 \nu_{0}}, \\
f\left(\xi=\xi_{0}\right)=0, \quad \xi_{0}=\frac{3 \nu(3-\nu)}{(2-\nu)(1+3 \nu)}, \\
f\left(\xi \rightarrow \xi_{+\infty} \rightarrow+\infty, \quad \xi_{+\infty}=\frac{3 \nu_{0}\left(3-\nu_{0}\right)}{\left(2-\nu_{0}\right)\left(1+3 \nu_{0}\right)} .\right.
\end{gathered}
$$

The function $f(\xi)$ is not defined for $\xi$ in the interval between $\xi_{0}$ and $\xi_{+\infty}$ since the base of the second factor on the rhs of eq. B.11 has to be positive for a real exponent.

We calculate the derivative of $E(\xi)$ from eq. B.11:

$$
\frac{d E}{d \xi}=E \frac{10}{w^{3}}\left\{\frac{\left(\nu-\nu_{0}\right) w}{6(1-\xi)}\left[\frac{w+3-7 \xi}{\left(\nu_{2}-\nu_{0}\right)\left(\nu_{2}-\nu\right)}-\frac{w-(3-7 \xi)}{\left(\nu_{1}-\nu_{0}\right)\left(\nu_{1}-\nu\right)}\right]+(3-7 \xi) \ln \left(\frac{\nu_{2}-\nu}{\nu_{2}-\nu_{0}} \frac{\nu_{1}-\nu_{0}}{\nu_{1}-\nu}\right)\right\} .
$$

A closer look at this function shows that for $0 \leq \nu, \nu_{0}<\frac{1}{2}$ the derivative $\frac{d E}{d \xi}$ has the same sign as the factor $\nu-\nu_{0}$, so that $E(\xi)$ and $f(\xi)$ are either stricly increasing or strictly decreasing.

We conclude that eq. B.11 has exactly one solution on the real $\xi$ axis. This solution is located in the interval $\xi_{0} \leq \xi<+\infty$, if $\nu>\nu_{0}$. For the case $\nu<\nu_{0}$, we obtain the following result: If $\frac{E}{E_{0}}<\frac{1+3 \nu}{1+3 \nu_{0}}$, then eq. B.11 has a solution in the interval $-\infty<\xi \leq \xi_{0}$. If $\frac{E}{E_{0}}>\frac{1+3 \nu}{1+3 \nu_{0}}$, then it has a solution in the interval $\xi_{+\infty}<\xi \leq+\infty$. Of course physically meaningful is only a solution in the interval $0 \leq \xi \leq 1$. Other results occur as a consequence of measurement errors in $\nu_{0}, \nu, E_{0}$ and $E$.

For numerical computations eq. B.10 should be used instead of B.11 to avoid overflow. 


\section{APPENDIX C: THE SC AND NI CRACK THEORIES}

In this appendix we present the corresponding formulas of O'Connell and Budiansky's self-consistent (SC) and Kachanov's non-interacting (NI) crack theory for comparison with the DEM theory. The equations are used in the main text for forward modeling (Figs. 6, 7) and inversion (Fg. 12). The model is a mixture of two sets of randomly oriented cracks: a fraction $\xi$ of wet cracks saturated with an incompressible fluid and a fraction $1-\xi$ of dry cracks.

The NI method calculates the change in potential energy by using the elastic moduli of the unfractured medium, while the SC method uses the moduli of the completely fractured medium. As a consequence there is a certain symmetry in the formulas for crack density and fluid saturation when moduli $E_{0}, \nu_{0}$ are interchanged with $E, \nu$.

\section{SC crack theory}

The elastic moduli are given by eqs. 17 of O'Connell \& Budiansky (1974),

$$
\begin{gathered}
\frac{K}{K_{0}}=1-\frac{16}{9} \frac{1-\nu^{2}}{1-2 \nu}(1-\xi) \epsilon, \\
\frac{E}{E_{0}}=1-\frac{16}{45} \frac{1-\nu^{2}}{2-\nu}[4+3(1-\xi)(2-\nu)] \epsilon, \\
\frac{\mu}{\mu_{0}}=1-\frac{32}{45} \frac{1-\nu}{2-\nu}[3+(1-\xi)(2-\nu)] \epsilon,
\end{gathered}
$$

and crack density by their eq. 18 ,

$$
\left.\epsilon=\frac{45}{16} \frac{(2-\nu)\left(\nu-\nu_{0}\right)}{\left(1-\nu^{2}\right)\left[2\left(1-2 \nu_{0}\right)-(1-\xi)\left(1+3 \nu_{0}\right)(2-\nu)\right]} \cdot\right\rangle
$$

By substituting $\epsilon$ on the rhs of eq. (C.2) for Young's modulus we obtain for the fluid saturation fraction

$$
\xi=\frac{3 \nu(3-\nu)-\frac{E}{E_{0}}\left[10 \nu_{0}-\left(1+3 \nu_{0}\right) \nu\right]}{(2-\nu)\left[1+3 \nu-\left(1+3 \nu_{0}\right) \frac{E}{E_{0}}\right]}
$$

and if we substitute this result for $\xi$ on the rhs of eq. C. 4 then we obtain for crack density

$$
\epsilon=\frac{9}{32} \frac{2-\nu}{1-\nu^{2}} \frac{(1+3 \nu) E_{0}-\left(1+3 \nu_{0}\right) E}{E_{0}} .
$$

If we write the moduli as functions of $P$ and $S$ wave velocities then we obtain eq. 62 of Budiansky $\&$ O'Connell (1976). The inverse problem to determine fluid saturation $\xi$ and crack density $\epsilon$ from measured data $\nu_{0}, E_{0}, \nu, E$ is solved by eqs. (C.5) and (C.6).

The $P$ wave modulus follows from (C.1) and (C.3) with eq. (18):

$$
\frac{K+\frac{4}{3} \mu}{K_{0}+\frac{4}{3} \mu_{0}}=1-\frac{16}{27} \frac{1+\nu_{0}}{1-\nu_{0}} \frac{1-\nu^{2}}{1-2 \nu}(1-\xi) \epsilon-\frac{64}{135} \frac{1-2 \nu_{0}}{1-\nu_{0}} \frac{1-\nu}{2-\nu}[3+(1-\xi)(2-\nu)] \epsilon \text {. }
$$

Poisson's ratio $\nu$ of the fractured medium is needed to compute elastic moduli as functions of $\epsilon$ and $\xi$. It is obtained as one of the roots of a cubic equation which follows from (C.4). 


\section{NI crack theory}

Kachanov's NI theory (Kachanov 1992) leads for Young's modulus and for the shear modulus to

$$
\begin{aligned}
& \frac{E_{0}}{E}=1+\frac{16}{45} \frac{1-\nu_{0}^{2}}{2-\nu_{0}}\left[4+3(1-\xi)\left(2-\nu_{0}\right)\right] \epsilon, \\
& \frac{\mu_{0}}{\mu}=1+\frac{32}{45} \frac{1-\nu_{0}}{2-\nu_{0}}\left[3+(1-\xi)\left(2-\nu_{0}\right)\right] \epsilon .
\end{aligned}
$$

Poisson's ratio $\nu$ is then given by

$$
\nu=\frac{\nu_{0}+\frac{16}{45} \frac{1-\nu_{0}^{2}}{2-\nu_{0}}\left[2-(1-\xi)\left(2-\nu_{0}\right)\right] \epsilon}{1+\frac{16}{45} \frac{1-\nu_{0}^{2}}{2-\nu_{0}}\left[4+3(1-\xi)\left(2-\nu_{0}\right)\right] \epsilon}
$$

and we obtain for the crack density

$$
\epsilon=\frac{45}{16} \frac{\left(2-\nu_{0}\right)\left(\nu-\nu_{0}\right)}{\left(1-\nu_{0}^{2}\right)\left[2(1-2 \nu)-(1-\xi)(1+3 \nu)\left(2-\nu_{0}\right)\right]} .
$$

By substituting this result in the rhs of eq. (C.8) for Young's modulus we obtain the saturation

$$
\xi=\frac{3 \nu_{0}\left(3-\nu_{0}\right)-\frac{E_{0}}{E}\left[10 \nu-(1+3 \nu) \nu_{0}\right]}{\left(2-\nu_{0}\right)\left[1+3 \nu_{0}-(1+3 \nu) \frac{E_{0}}{E}\right]}
$$

and then for the crack density

$$
\epsilon=\frac{9}{32} \frac{2-\nu_{0}}{1-\nu_{0}^{2}} \frac{(1+3 \nu) E_{0}-\left(1+3 \nu_{0}\right) E}{E} .
$$

We calculate the $P$ wave modulus by using (C.10)

$$
\frac{K_{0}+\frac{4}{3} \mu_{0}}{K+\frac{4}{3} \mu}=\frac{\mu_{0}}{\mu} \frac{1-\nu_{0}}{1-2 \nu_{0}} \frac{1-2 \nu}{1-\nu}=\frac{\mu_{0}}{\mu} \frac{1+\frac{16}{9} \frac{1-\nu_{0}^{2}}{1-2 \nu_{0}}(1-\xi) \epsilon}{1+\frac{32}{45} \frac{1+\nu_{0}}{2-\nu_{0}}\left[1+2(1-\xi)\left(2-\nu_{0}\right)\right] \epsilon},
$$

where the shear modulus is given by eq. (C.9).

By using eq. (C.10) the $P$ wave modulus can be written as

$$
\frac{K_{0}+\frac{4}{3} \mu_{0}}{K+\frac{4}{3} \mu}=1+\frac{16}{27} \frac{1+\nu}{1-\nu} \frac{1-\nu_{0}^{2}}{1-2 \nu_{0}}(1-\xi) \epsilon+\frac{64}{135} \frac{1-2 \nu}{1-\nu} \frac{1-\nu_{0}}{2-\nu_{0}}\left[3+(1-\xi)\left(2-\nu_{0}\right)\right] \epsilon,
$$

which is a similar form as the corresponding equation (C.7) of the SC theory.

Eqs. (C.9) and (C.14) can be used for forward modeling and eqs. (C.12) and (C.13) for the inverse problem. 